\title{
On Subtree Number Index of Generalized Book Graphs, Fan Graphs, and Wheel Graphs
}

\author{
Daoqiang Sun, ${ }^{1}$ Zhengying Zhao, ${ }^{1}$ Xiaoxiao Li, ${ }^{1}$ Jiayi Cao, ${ }^{2}$ and Yu Yang $\mathbb{D D}^{1,3}$ \\ ${ }^{1}$ School of Software, Pingdingshan University, Pingdingshan 467000, China \\ ${ }^{2}$ Navigation College, Dalian Maritime University, Dalian 116026, China \\ ${ }^{3}$ School of Mathematical Sciences, Shanghai Jiao Tong University, Shanghai 200240, China \\ Correspondence should be addressed to Yu Yang; yangyu@sjtu.edu.cn
}

Received 23 January 2021; Revised 27 February 2021; Accepted 4 March 2021; Published 10 April 2021

Academic Editor: Ahmet Sinan Cevik

Copyright (c) 2021 Daoqiang Sun et al. This is an open access article distributed under the Creative Commons Attribution License, which permits unrestricted use, distribution, and reproduction in any medium, provided the original work is properly cited.

With generating function and structural analysis, this paper presents the subtree generating functions and the subtree number index of generalized book graphs, generalized fan graphs, and generalized wheel graphs, respectively. As an application, this paper also briefly studies the subtree number index and the asymptotic properties of the subtree densities in regular book graphs, regular fan graphs, and regular wheel graphs. The results provide the basis for studying novel structural properties of the graphs generated by generalized book graphs, fan graphs, and wheel graphs from the perspective of the subtree number index.

\section{Introduction}

The invariants of the graph and its topological indices have important significance in studying the properties of chemical molecular graphs and network graphs [1-4]. The Wiener index, for example, is defined as the sum of the distances of all disordered vertex pairs, and it can be used to estimate the boiling point of the alkanes $[5,6]$. Therefore, new topological indices are constantly introduced by worldwide scholars in recent years.

As a representative structural based index, the subtree number index $\operatorname{STN}(G)$ of a graph $G$ (also known as $\rho$-index $[7,8])$, which is defined as the number of nonempty labeled subtrees of $G$, plays an important role in areas such as biological reconstruction [9], reliable network construction [10], and machine learning [11] and therefore has attracted much attention in recent years.

The subtree number problem was first proposed by Jamison, when studying the subtree average order of trees $[12,13]$. Székely and Wang [8] characterized the extremal graph among all $n$ vertices tree. Meanwhile, they presented the subtree number formula of good binary tree and relation between the Wiener index and subtree number index of trees. Yan and Yeh [14] proposed a linear algorithm for enumerating subtrees of trees and further characterized the trees with the second to fifth largest numbers of subtrees and the tree with the second minimum number of subtrees through graph transformation and generating function. With generating function and cycle contraction carrying weights technique, Yang et al. [15-17] solved the subtree enumeration problem and characterized the extremal graphs among all hexagonal, phenylene chains, and spiro, polyphenyl hexagonal chains of length $n$, respectively.

Using the generating function method, more recently, Chin et al. [18] studied the enumeration problem of subtrees and spanning subtrees of complete graphs, complete bipartite graphs, and theta graphs. Yang et al. [17] studied the expected subtree number index in random polyphenylene and spiro chains. By introducing new "middle parts" of a tree, Li et al. [19] studied several extreme ratios of distance and subtree problems in binary trees. With the combinatorial technique, Kamiński and Prałat [20] provided the upper and lower bounds for the number of subtrees of random tree. Zhang et al. [21] presented the concepts of the eccentric subtree number and studied some extremal results with respect to this topological index in a tree.

Wheel graphs, fan graphs, and book graphs are three representative cyclic graphs. Various properties of these 
graphs have been studied, for example, Lin et al. [22] studied the Laplacian spectral features of vertex extension of wheel graphs and multifan graphs. Daoud [23] studied the number of spanning trees of wheel graphs and complex graphs generated by wheel graphs. Yang et al. [24] studied the subtree problem of wheel graphs and multifan graphs with the generating functions. Barioli [25] studied the complete matrix of book graphs. Based on wheel graph and chicken swarm optimization, Yu et al. [26] proposed a novel hybrid localization scheme for deep mine. Liu et al. [27] proved that multifan graphs were determined by their Laplacian spectra.

We first introduce related terminologies and notations in Section 2. The subtree generating functions and subtree number indices of generalized book graphs $B(n)(n \geq 2)$, fan graphs $F(n)(n \geq 3)$, and wheel graphs $W(n)(n \geq 3)$ are derived in Section 3. Some special cases are also discussed as immediate consequences. In Section 4, we analyze the asymptotic characteristics of the subtree densities of the aforementioned generalized graphs. We summarize our work in Section 5.

\section{Terminology and Notation}

Let $G=(V(G), E(G) ; f, g)$ be a weighted graph with vertex set $V(G)=\left\{v_{1}, v_{2}, \ldots, v_{n}\right\}$, edge set $E(G)=\left\{e_{1}, e_{2}, \ldots, e_{m}\right\}$, vertex weight function $f$, and edge weight function $g$. Unless otherwise stated, we specify $f: V(G) \longrightarrow \Re$ and $g: E(G) \longrightarrow \mathfrak{R}$, where $\mathfrak{R}$ is a commutative ring with a unit element 1 . In what follows, we list the symbols that will be used in later discussion.

(i) Denote by $L(G)$ the leaf set of $G$.

(ii) Denote by $G \backslash X$ the graph obtained from $G$ by removing $X$ from $G$.

(iii) Denote by $S(G)$ the set of all subtrees of $G$.

(iv) Denote by $K_{1, n}$ a star with $n+1$ vertices and $P_{n}$ a path with $n$ vertices.

(v) Denote by $S(G ; X)$ the set of subtrees of $G$ containing $X$, where $X$ can be a vertex or an edge set.

(vi) Denote by $S\left(G ; v, P_{n}\right)$ the set of subtrees of $G$ containing both vertex $v$ and path $P_{n}$.

For any subtree $T \in S(G)$, the weight of $T$ is defined as

$$
w(T)=\prod_{v \in V(T), e \in E(T)} f(v) g(e),
$$

and the subtree generating function of $G$ is defined as $F(G ; f, g)=\sum_{T_{s} \in S(G)} w\left(T_{s}\right)$. Similarly, we define $F(G ; f, g$; $X)=\sum_{T_{s} \in S(G ; X)} w\left(T_{s}\right)$, the subtree generating function of $G$ containing $X$, and $F\left(G ; f, g ; v, P_{n}\right)=\sum_{T_{s} \in S\left(G ; v, P_{n}\right)} w\left(T_{s}\right)$, the subtree generating function of $G$ containing both vertex $v$ and path $P_{n}$.

Letting vertex and edge weight $f=g=1$ in the above generating functions, we have the corresponding subtree number indices, namely, $\operatorname{STN}(G)=F(G ; 1,1)$, $\operatorname{STN}(G$; $X)=F(G ; 1,1 ; X)$, and $\operatorname{STN}\left(G ; v, P_{n}\right)=F\left(G ; 1,1 ; v, P_{n}\right)$.

Next, we introduce some lemmas and definitions that will be used in our arguments. Let $T=(V(T), E(T) ; f, g)$ be a weighted tree on $n$ vertices, $v_{i}$ be a vertex of $T, u \neq v_{i}$ be a pendant vertex, and $e=(u, v)$ be the pendant edge of $T$. We define a weighted tree $T^{\prime}=\left(V\left(T^{\prime}\right), E\left(T^{\prime}\right) ; f^{\prime}, g^{\prime}\right)$ from $T$ as follows: $V\left(T^{\prime}\right)=V(T) \backslash\{u\}, E\left(T^{\prime}\right)=E(T) \backslash\{e\}$, and

$$
f^{\prime}\left(v_{s}\right)= \begin{cases}f(v)(f(u) g(e)+1), & \text { if } v_{s}=v, \\ f\left(v_{s}\right), & \text { otherwise, }\end{cases}
$$

for any $v_{s} \in V\left(T^{\prime}\right), g^{\prime}(e)=g(e)\left(e \in E\left(T^{\prime}\right)\right)$.

Lemma 1 (see [14]). With the above notations, we have

$$
\begin{aligned}
F\left(T ; f, g ; v_{i}\right) & =F\left(T^{\prime} ; f^{\prime}, g^{\prime} ; v_{i}\right), \\
F(T ; f, g) & =F\left(T^{\prime} ; f^{\prime}, g^{\prime}\right)+f(u)
\end{aligned}
$$

Definition 1. If the $n$ pendant edges of the star $K_{1, n}$ are replaced by paths of length $l_{i}(i=1,2, \ldots, n)$, the resulting tree is called a generalized star tree $K\left(l_{1}, l_{2}, \ldots, l_{n}\right)$ or simply $K(n)$.

Definition 2. Define $B\left(l_{0}, l_{1}, \ldots, l_{n}\right)$ (or simply $B(n)(n \geq 2)$ ) the $n$-page generalized book graph constructed by joining the end vertices $u_{i}$ and $v_{i}$ of the $n+1$ internally disjoint paths $u_{i} w_{i, 1} w_{i, 2} \ldots w_{i, l_{i}} v_{i}(i=0,1, \ldots, n)$ of length $l_{i}+1$, where $u_{0}=$ $u_{1}=\cdots=u_{n}=u$ and $v_{0}=v_{1}=\cdots=v_{n}=v$. Obviously, $B(n)$ has $\left(2+\sum_{i=0}^{n} l_{i}\right)$ vertices and $\sum_{i=0}^{n}\left(l_{i}+1\right)$ edges.

Definition 3. Define $F\left(l_{1}, \ldots, l_{n} ; m_{1}, \ldots, m_{n-1}\right)$ (or simply $F(n)(n \geq 3))$ the generalized fan graph obtained by firstly constructing a generalized star through identifying the end vertices $v_{i, 0}(i=1,2, \ldots, n)$ of the $n$ internally disjoint paths $v_{i, 0} v_{i, 1} v_{i, 2} \ldots v_{i, l_{i}} c_{i}\left(l_{i} \geq 0\right)$ together, where $v_{i, 0}=c_{0}$ and then connecting vertex pair $c_{i}, c_{i+1}$ with path $c_{i} v_{i, 1}^{*} v_{i, 2}^{*} \ldots v_{i, m_{i}}^{*} c_{i+1}$ $(i=1,2, \ldots, n-1)$ of length $m_{i}+1\left(m_{i} \geq 0\right)$, where $v_{i, 0}=c_{0}$, $l_{i} \geq 0(i=1,2, \ldots, n)$, and $m_{i} \geq 0(i=1,2, \ldots, n-1)$ (specifying that $\left.m_{0}=0\right)$. For brevity, we abbreviate some notations.

(i) Denote $\bar{e}_{i, 1}=\left(c_{0}, v_{i, 1}\right), \quad \bar{e}_{i, j}=\left(v_{i, j-1}, v_{i, j}\right), \quad \bar{e}_{i, l_{i}+1}=$ $\left(v_{i, l_{i}}, c_{i}\right)\left(i=1, \ldots, n ; j=2, \ldots, l_{i}\right)$.

(ii) Denote $e_{i, 1}=\left(c_{i}, v_{i, 1}^{*}\right), \quad e_{i, j}=\left(v_{i, j-1}^{*}, v_{i, j}^{*}\right), e_{i, m_{i}+1}=$ $\left(v_{i, m_{i}}^{*}, c_{i+1}\right)\left(i=1, \ldots, n-1 ; j=2, \ldots, m_{i}\right)$, specifying that $e_{0, i}=\varnothing$.

Definition 4. Define $W\left(l_{1}, \ldots, l_{n} ; m_{1}, \ldots, m_{n}\right)$ (or simply $W(n)(n \geq 3))$ the generalized wheel graph constructed from the generalized fan graph $F(n)$ (see Definition 3) by joining vertices pair $c_{1}$ and $c_{n}$ of the generalized fan graph $F(n)$ with a path $v_{n, 0}^{*} v_{n, 1}^{*} \ldots v_{n, m_{n}}^{*} v_{n, m_{n}+1}^{*}$, where $v_{n, 0}^{*}=c_{n}$ and $v_{n, m_{n}+1}^{*}=c_{1}$. Similarly, we abbreviate some notations for the brevity.

(i) Denote $\tilde{e}_{i, 1}=\left(c_{0}, v_{i, 1}\right), \quad \tilde{e}_{i, j}=\left(v_{i, j-1}, v_{i, j}\right), \quad \tilde{e}_{i, l_{i}+1}$ $=\left(v_{i, l_{i}}, c_{i}\right)\left(i=1, \ldots, n ; j=2, \ldots, l_{i}\right)$.

(ii) Denote $e_{i, 1}^{*}=\left(c_{i}, v_{i, 1}^{*}\right), \quad e_{i, j}^{*}=\left(v_{i, j-1}^{*}, v_{i, j}^{*}\right), \quad e_{i, m_{i}+1}^{*}$ $=\left(v_{i, m_{i}}^{*}, c_{i+1}\right),\left(i=1, \ldots, n ; j=2, \ldots, m_{i}\right)$, specifying that $e_{0, i}^{*}=\varnothing$. 


\section{Subtree Generating Functions for Generalized Book Graphs, Fan Graphs, and Wheel Graphs}

3.1. Subtree Generating Function for Generalized Book Graphs. Before deriving the subtree generating function for generalized book graphs, we first introduce some lemmas.

Lemma 2 (see [14]). Let $P_{n}=\left(V\left(P_{n}\right), E\left(P_{n}\right) ; f, g\right)$ be a weighted path on $n$ vertices, with $V\left(P_{n}\right)=\left\{v_{0}, v_{1}, \ldots, v_{n-1}\right\}$, $E\left(P_{n}\right)=\left\{\left(v_{i}, v_{i+1}\right) \mid i=0,1, \ldots, n-2\right\}, f(v)=y\left(v \in V\left(P_{n}\right)\right)$ and $g(e)=z\left(e \in E\left(P_{n}\right)\right)$; then,

$$
\begin{aligned}
F\left(P_{n} ; f, g\right) & =\sum_{i=0}^{n-1}(n-i) y^{i+1} z^{i}, \\
F\left(P_{n} ; f, g ; v_{0}\right) & =\sum_{i=0}^{n-1} y^{i+1} z^{i} .
\end{aligned}
$$

Lemma 3 (see [15]). Let $G^{\prime}=\left(V\left(G^{\prime}\right), E\left(G^{\prime}\right) ; f, g\right)$ be a weighted graph with the common edge $(u, v)$, $f(v)=y\left(v \in V\left(G^{\prime}\right)\right), g(e)=z\left(e \in E\left(G^{\prime}\right)\right), V\left(G^{\prime}\right)=V\left(G_{1}^{\prime}\right)$ $\cup V\left(G_{2}^{\prime}\right)$, and $E\left(G^{\prime}\right)=E\left(G_{1}^{\prime}\right) \cup E\left(G_{2}^{\prime}\right), \quad G_{1}^{\prime} \cap G_{2}^{\prime}=(u, v)$, where $G_{1}^{\prime}, G_{2}^{\prime}$ are two weighted graphs; then,

$$
\begin{aligned}
F\left(G^{\prime} ; f, g\right)= & \frac{F\left(G_{1}^{\prime} ; f, g ;(u, v)\right) F\left(G_{2}^{\prime} ; f, g ;(u, v)\right)}{y^{2} z} \\
& +F\left(G^{\prime} \backslash(u, v) ; f, g\right) .
\end{aligned}
$$

Lemma 4 (see [16]). Let $U_{n}=\left(V\left(U_{n}\right), E\left(U_{n}\right) ; f, g\right)$ be a weighted unicyclic graph with $V\left(U_{n}\right)=\left\{v_{1}, v_{2}, \ldots, v_{n}\right\}$, $E\left(U_{n}\right)=\left\{\left(v_{i}, v_{i+1}\right) \mid i=1,2, \ldots, n\right\}\left(\right.$ where $\left.\quad v_{n+1}=v_{1}\right)$, $f(v)=y\left(v \in V\left(U_{n}\right)\right)$, and $g(e)=z\left(e \in E\left(U_{n}\right)\right)$, respectively; then, for any fixed vertex $v_{i} \in V\left(U_{n}\right)$ and any continuous $k$ edge set $\cup_{j=1}^{k}\left(v_{i+j-1}, v_{i+j}\right)(k=1,2, \ldots, n-1)$, we have

$$
\begin{array}{r}
F\left(U_{n} ; f, g ; v_{i}\right)=\sum_{i=1}^{n} i y^{i} z^{i-1}, \\
F\left(U_{n} ; f, g ; \bigcup_{j=1}^{k}\left(v_{i+j-1}, v_{i+j}\right)\right)=\sum_{i=1}^{n-k} i y^{i+k} z^{i+k-1} .
\end{array}
$$

The following Lemma follows from Lemmas 1 and 2 .

Lemma 5. Let $K(n)=(V(K(n)), E(K(n)) ; f, g)$ be a weighted generalized star tree, with $f(v)=y(v \in V(K(n)))$ and $g(e)=z(e \in E(K(n)))$; then,

$$
F(K(n) ; f, g)=\frac{1}{y^{n-1}} \prod_{i=1}^{n} \sum_{j=1}^{l_{i}+1} y^{j} z^{j-1}+\sum_{i=1}^{n} \sum_{j=1}^{l_{i}}\left(l_{i}-j+1\right) y^{j} z^{j-1} \text {. }
$$

Theorem 1. Let $B(n)=(V(B(n)), E(B(n)) ; f, g)$ be an $n$-page weighted generalized book graph, with $f(v)=y(v \in V(B(n)))$ and $g(e)=z(e \in E(B(n)))$; then,

$$
F(B(n) ; f, g)=\sum_{i=0}^{n} \frac{\left(y^{l_{i}+2} z^{l_{i}+1}\right)^{1-n}}{\sum_{k=1}^{l_{i}+1}\left(k y^{k+l_{i}+1} z^{k+l_{i}}\right)} \prod_{j=0}^{n} \sum_{k=1}^{l_{j}+1}\left(k y^{k+l_{i}+1} z^{k+l_{i}}\right)+\frac{2}{y^{n}} \prod_{i=0}^{n} \sum_{j=1}^{l_{i}+1} y^{j} z^{j-1}+\sum_{i=0}^{n} \sum_{j=1}^{l_{i}}\left(l_{i}-j+1\right) y^{j} z^{j-1}
$$

Proof. We divide the subtrees of generalized book graph $B(n)$ into four categories:

(1) Containing $u$ but not $v$.

(2) Containing $v$ but not $u$.

(3) Containing neither $u$ nor $v$.

(4) Containing both $u$ and $v$.

With structural analysis and Lemma 5, we have the subtree generating function of cases (1) and (2) as

$$
\frac{1}{y^{n}} \prod_{i=0}^{n} \sum_{j=1}^{l_{i}+1} y^{j} z^{j-1}
$$

With Lemma 1 , it is easy to see that the subtree generating function of case (3) is

$$
\sum_{i=0}^{n} \sum_{j=1}^{l_{i}}\left(l_{i}-j+1\right) y^{j} z^{j-1}
$$

Denote by $P_{u v}^{i}=u w_{i, 1} w_{i, 2} \ldots w_{i, l_{i}} v(i=0,1,2, \ldots, n)$ the path connecting the vertex pair $u, v$, and $U_{l_{i}+l_{j}+2}^{i, j}=P_{u v}^{i} w_{j, l_{j}} \ldots w_{j, 2} w_{j, 1} u(j \neq i, j \in\{0,1, \ldots, n\}) \quad$ an unicyclic graph. With structural analysis, equation (8) in Lemma 4, and Lemma 3, we have the subtree generating function of case (4) as

$$
\sum_{i=0}^{n} \frac{\prod_{0 \leq j \neq i \leq n} F\left(U_{l_{i}+l_{j}+2}^{i, j} ; f, g ; P_{u v}^{i}\right)}{\left(y^{l_{i}+2} z^{l_{i}+1}\right)^{n-1}} .
$$

The theorem follows from equations (11)-(13).

We can further obtain the subtree generating function of special generalized book graphs.

Definition 5. Let $B\left(l_{0}, l_{1}, \ldots, l_{n}\right)$ be an $n$-page generalized book graph and $k$ be a positive integer; we call $B\left(l_{0}, l_{1}, \ldots, l_{n}\right)$ an $n$-page $k$ regular book graph, denoted by 
$B_{n, k}(n \geq 2)$, if the lengths of its $n+1$ internally disjoint paths are all equal to $k$ (namely, $\left.l_{i}=k(i=0,1, \ldots, n)\right)$. Obviously, $B_{n, k}(n \geq 2)$ has $(n+1) k+2$ vertices and $(n+1)(k+1)$ edges.

With Theorem 1, it is not difficult to obtain the following corollary.

Corollary 1. Let $B_{n, k}=\left(V\left(B_{n, k}\right), E\left(B_{n, k}\right) ; f, g\right)$ be an n-page $k$ regular book graph, with $f(v)=y\left(v \in V\left(B_{n, k}\right)\right)$ and $g(e)=z\left(e \in E\left(B_{n, k}\right)\right)$; then,

$$
\begin{aligned}
F\left(B_{n, k} ; f, g\right)= & (n+1) \sum_{i=0}^{k-1}(k-i) y^{i+1} z^{i}+\frac{2}{y^{n}}\left(\sum_{i=1}^{k+1} y^{i} z^{i-1}\right)^{n+1} \\
& +\frac{n+1}{\left(y^{k+2} z^{k+1}\right)^{n-1}}\left(\sum_{i=1}^{k+1} i y^{i+k+1} z^{i+k}\right)^{n} .
\end{aligned}
$$

By substituting $y=1, z=1$ into equations (10) and (14), we have the corresponding subtree number indices.

Corollary 2. The subtree number index of the n-page generalized book graph $B(n)$ is

$$
\begin{aligned}
\operatorname{STN}(B(n))= & 2 \prod_{i=0}^{n}\left(l_{i}+1\right) \\
& +\sum_{i=0}^{n} \sum_{j=0}^{l_{i}-1}\left(l_{i}-j\right)+\sum_{i=0}^{n} \frac{\prod_{j=0}^{n}\left(l_{j}+1\right)\left(l_{j}+2\right)}{2^{n}\left(l_{i}+1\right)\left(l_{i}+2\right)} .
\end{aligned}
$$

Corollary 3. The subtree number index of the n-page $k$ regular book graph $B_{n, k}$ is

$$
\begin{aligned}
\operatorname{STN}\left(B_{n, k}\right)= & (n+1) \frac{k(k+1)}{2}+2(k+1)^{n+1} \\
& +(n+1) \frac{\left(k^{2}+3 k+2\right)^{n}}{2^{n}} .
\end{aligned}
$$

With Corollary 3, we have the subtree number index of the $B_{n, k}$ as illustrated in Table 1 .

3.2. Subtree Generating Function of Two-Tailed Generalized Fan Graph. Before solving the subtree generating functions of generalized fan graphs and generalized wheel graphs, we firstly introduce and solve the subtree generating function computing problem of the two-tailed generalized fan graphs. Firstly, we employ the convention that $\cup_{t=i}^{j} \bar{e}_{t, 1}=\varnothing(j<i)$.

Definition 6. Denote by $\bar{F}_{n, i}(i=1,2,3, \ldots, n)$ the generalized fan graph of $F(n) \backslash\left(e_{i-1, m_{i-1}+1} \cup \cup_{j=1}^{i-1} \bar{e}_{j, 1}\right)$ that contains $c_{0}$ and $c_{i}$; moreover, define $P_{c_{i}, c_{n}}$ the path tree of $\bar{F}_{n, i} \backslash\left(\cup_{r=i}^{n} \bar{e}_{r, l_{r}+1}\right)$ that contains vertex $c_{i}$.

With structural analysis and equations (9) and (13), we can obtain the following lemma.

Lemma 6. Keeping above symbols and definitions, we have

$$
F\left(\bar{F}_{n, i} ; f, g ; c_{0}, P_{c_{i}, c_{n}}\right)=\sum_{t=i}^{n} \frac{(y z)^{l_{t}+1+\sum_{j=i}^{n-1}\left(m_{j}+1\right)}}{y^{n-i-1} \sum_{p=1}^{l_{t}+1} p y^{p} z^{p-1}} \prod_{j=i}^{n} \sum_{p=1}^{l_{j}+1} p y^{p} z^{p-1} .
$$

Definition 7. Define $\widetilde{F}_{a, b}^{i}\left(l_{i}, \ldots, l_{n} ; m_{i}, \ldots, m_{n-1}\right)$ (or simply $\left.\widetilde{F}_{a, b}^{n, i}(i=1, \ldots, n)\right)$ the two-tailed generalized fan graph obtained from $\bar{F}_{n, i}(i=1,2,3, \ldots, n)$ by attaching a path $P_{a+1}{ }^{\prime}=$ $v_{0}^{\prime} v_{1}^{\prime} v_{2}^{\prime} \ldots v_{a}^{\prime}$ to vertex $c_{i}$ and path $P_{b+1}^{\prime \prime}=v_{o}^{\prime \prime} v_{1}^{\prime \prime} v_{2}^{\prime \prime} \ldots v_{b}^{\prime \prime}$, to vertex $c_{n}$, respectively, where $c_{i}=v_{0}^{\prime}$ and $c_{n}=v_{0}^{\prime \prime}$ (see Figure 1 for an illustration).

(i) Denote by $F_{a, b}^{n, i}(i=1,2, \ldots, n)$ the $k$ regular twotailed generalized fan graph with $l_{i}=m_{j}=k$ $(i=1, \ldots, n ; j=1, \ldots, n-1 ; k \geq 0)$.

(ii) Denote by $\widetilde{T}_{a, b}^{n, i}=\widetilde{F}_{a, b}^{n, i} \backslash c_{0}$ and $T_{a, b}^{n, i}=F_{a, b}^{n, i} \backslash$ $c_{0}(i=1,2,3, \ldots, n)$ the two weighted trees.

Lemma 7. Let $\widetilde{F}_{a, b}^{n, i}=\left(V\left(\widetilde{F}_{a, b}^{n, i}\right), E\left(\widetilde{F}_{a, b}^{n, i}\right) ; f, g\right)(i=1,2, \ldots$, $n)$ be a weighted two-tailed generalized fan graph, with $f(v)=y\left(v \in V\left(\widetilde{F}_{a, b}^{n, i}\right)\right)$ and $g(e)=z\left(e \in E\left(\widetilde{F}_{a, b}^{n, l}\right)\right)$, respectively; then,

$$
\begin{aligned}
F\left(\widetilde{F}_{a, b}^{n, i} ; f, g ; c_{0}\right)= & \frac{1}{y^{2}} \sum_{t=i}^{n-1} F\left(\bar{F}_{n, t+1} ; f, g ; c_{0}, P_{c_{t+1}, c_{n}}\right) \sum_{j=t / n-1\rfloor}^{m_{t}} F\left(\widetilde{F}_{a, m_{t}-j}^{t, i} ; f, g ; c_{0}\right)(y z)^{j} \\
& \cdot \sum_{j=1}^{b+1} y^{j} z^{j-1}+\sum_{k=1}^{a+1}(y z)^{k-1} F\left(\bar{F}_{n, i} ; f, g ; c_{0}, P_{c_{i}, c_{n}}\right) \sum_{k=1}^{b+1}(y z)^{k-1}+F\left(\widetilde{F}_{a, m_{n-1}}^{n-1, i} ; f, g ; c_{0}\right) \sum_{k=1}^{l_{n}+b+2}(y z)^{k-1},
\end{aligned}
$$

with $F\left(\bar{F}_{n, i} ; f, g ; c_{0}, P_{c_{i}, c_{n}}\right)$ as in equation (17) and $F(\widetilde{F}$ $\left.{ }_{a, b} ; f, g ; c_{0}\right)=\sum_{t=1}^{l_{i}+1} y^{t} z^{t-1}+y^{l_{i}} z^{l_{i}+1} \sum_{t=1}^{a+1} y^{t} z^{t-1} \sum_{t=1}^{b+1} y^{t} z^{t-1}$.
Proof. We categorize the subtrees of $\widetilde{F}_{a, b}^{n, i}$ containing $c_{0}$ into four cases: 
TABLE 1: The subtree number index $\operatorname{STN}\left(B_{n, k}\right)$ of the $n$-page $k$ regular book graph $B_{n, k}(k=1,2, \ldots, 5 ; n=2,3, \ldots, 11)$.

\begin{tabular}{|c|c|c|c|c|c|}
\hline$n$ & $\operatorname{STN}\left(B_{n, 1}\right)$ & $\operatorname{STN}\left(B_{n, 2}\right)$ & $\operatorname{STN}\left(B_{n, 3}\right)$ & $\operatorname{STN}\left(B_{n, 4}\right)$ & $\operatorname{STN}\left(B_{n, 5}\right)$ \\
\hline 2 & 46 & 171 & 446 & 955 & 1800 \\
\hline 3 & 144 & 1038 & 4536 & 14790 & 39696 \\
\hline 4 & 474 & 6981 & 52078 & 259425 & 988032 \\
\hline 5 & 1592 & 48132 & 608228 & 4587560 & 24598008 \\
\hline 6 & 5366 & 330987 & 7032810 & 79890695 & 600922824 \\
\hline 7 & 18016 & 2252634 & 80131120 & 1367656330 & 14412067680 \\
\hline 8 & 60082 & 15155937 & 900524342 & 23069921965 & 340425889776 \\
\hline 9 & 198888 & 100895088 & 10002097212 & 384453125100 & 7942921398312 \\
\hline 10 & 653646 & 665482263 & 110008388674 & 6343251953235 & 183479416354488 \\
\hline 11 & 2133968 & 4354627590 & 1200033554504 & 103797558593870 & 4203334360071504 \\
\hline
\end{tabular}

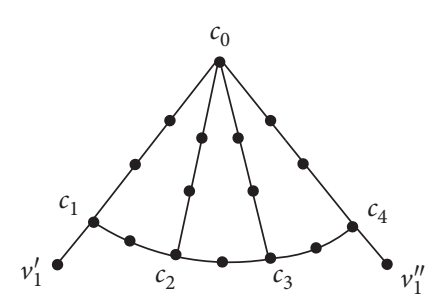

(a)

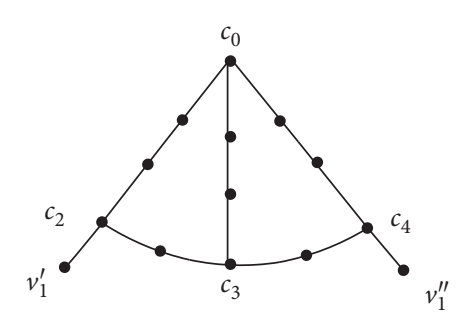

(b)

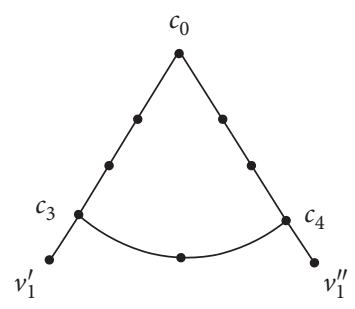

(c)

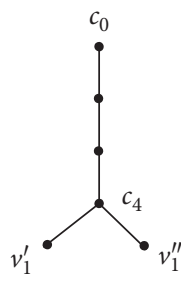

(d)

FIgURE 1: Two-tailed generalized fan graph $\widetilde{F}_{1,1}^{4, i}(i=1,2,3,4)$. (a) $\widetilde{F}_{1,1}^{4,1}$. (b) $\widetilde{F}_{1,1}^{4,2}$. (c) $\widetilde{F}_{1,1}^{4,3}$. (d) $\widetilde{F}_{1,1}^{4,4}$.

$$
S\left(\widetilde{F}_{a, b}^{n, i} ; c_{0}\right)=\bar{S}_{1} \cup \bar{S}_{2} \cup \bar{S}_{3} \cup \bar{S}_{4},
$$

where

(i) $\bar{S}_{1}$ is the set of subtrees in $S\left(\widetilde{F}_{a, b}^{n, i} ; c_{0}\right)$ that does not contain edge $e_{n-1, m_{n-1}+1}$.

(ii) $\bar{S}_{2}$ is the set of subtrees in $S\left(\widetilde{F}_{a, b}^{n, i} ; c_{0}\right)$ that contains edge set $\cup_{k=m_{n-1}+1-j}^{m_{n-1}+1} e_{n-1, k}, \quad$ but not edge $e_{n-1, m_{n-1}-j}\left(j=0,1, \ldots, m_{n-1}-1\right)$.

(iii) $\bar{S}_{3}$ is the set of subtrees in $S\left(\widetilde{F}_{a, b}^{n, i} ; c_{m_{n-1-p}+1}\right)$ that contains edge set $\cup_{k=n-p}^{n-1} \cup{ }_{j=1}^{m_{k}+1} e_{k, j} \cup \cup_{j=m_{n-1-p}+2-q}^{a, e_{n-1-p, j}}$, but not edge $e_{n-p-1, m_{n-1-p}-q+1}$ $\left(p=1,2, \ldots, n-i-1 ; q=0,1, \ldots, m_{n-p-1}\right)$.

(iv) $\bar{S}_{4}$ is the set of subtrees in $S\left(\widetilde{F}_{a, b}^{n, i} ; c_{0}\right)$ that contains edge set $\cup_{k=i}^{n-1} \cup_{j=1}^{m_{k}+1} e_{k, j}$.
For the case $\bar{S}_{1}$, we define path tree $\bar{P}_{l_{n}+b+2}=c_{0} v_{n, 1} v_{n, 2} \ldots v_{n, l_{n}} c_{n} P_{b+1}{ }^{\prime \prime}$, and it is not difficult to obtain

$$
\begin{aligned}
\sum_{T_{1} \in \bar{S}_{1}} w\left(T_{1}\right)= & \frac{1}{y} F\left(\bar{P}_{l_{n}+b+2} ; f, g ; c_{0}\right) \\
& \times F\left(\widetilde{F}_{a, m_{n-1}}^{n-1, i} ; f, g ; c_{0}\right)=F\left(\widetilde{F}_{a, m_{n-1}}^{n-1, i} ; f, g ; c_{0}\right) \sum_{j=1}^{l_{n}+b+2}(y z)^{j-1} .
\end{aligned}
$$

For the case $\bar{S}_{2}$, with structural analysis and equation (17), we obtain

$$
\begin{aligned}
\sum_{T_{2} \in \bar{S}_{2}} w\left(T_{2}\right)= & \frac{1}{y^{2}} \sum_{j=1}^{m_{n-1}}\left(F\left(\widetilde{F}_{a, m_{n-1}-j}^{n-1, i} ; f, g ; c_{0}\right) F\left(\bar{F}_{n, n} ; f, g ; c_{0}, P_{c_{n}, c_{n}}\right) \prod_{k=1}^{j} f\left(v_{n-1, m_{n-1}-k+1}^{*}\right) g\left(e_{n-1, m_{n-1}-k+2}\right) F\left(P_{b+1}^{\prime \prime} ; f, g ; c_{n}\right)\right) \\
& \frac{1}{y^{2}} \sum_{j=1}^{m_{n-1}} F\left(\widetilde{F}_{a, m_{n-1}-j}^{n-1, i} ; f, g ; c_{0}\right) y^{l_{n}+2} z^{l_{n}+1}(y z)^{j} \sum_{j=1}^{b+1} y^{j} z^{j-1} .
\end{aligned}
$$

Similar to analysis of case $\bar{S}_{2}$, we can obtain 


$$
\begin{aligned}
\sum_{T_{3} \in \bar{S}_{3}} w\left(T_{3}\right) & =\frac{1}{y^{2}} \sum_{t=i}^{n-2} F\left(\bar{F}_{n, t+1} ; f, g ; c_{0}, P_{c_{t+1}, c_{n}}\right)\left(\sum_{j=0}^{m_{t}} F\left(\widetilde{F}_{a, m_{t}-j}^{t, i} ; f, g ; c_{0}\right) \prod_{p=1}^{j} f\left(v_{t, m_{t}-p+1}^{*}\right) g\left(e_{t, m_{t}-p+2}\right) F\left(P_{b+1}^{\prime \prime} ; f, g ; c_{n}\right)\right) \\
& =\frac{1}{y^{2}} \sum_{t=i}^{n-2} F\left(\bar{F}_{n, t+1} ; f, g ; c_{0}, P_{c_{t+1}, c_{n}}\right) \sum_{j=0}^{m_{t}}(y z)^{j} F\left(\widetilde{F}_{a, m_{t}-j}^{t, i} ; f, g ; c_{0}\right) \sum_{k=1}^{b+1} y^{k} z^{k-1} .
\end{aligned}
$$

For the case $\bar{S}_{4}$, with equations (5) and (17), we can get

$$
\begin{aligned}
\sum_{T_{4} \in \bar{S}_{4}} w\left(T_{4}\right) & =\frac{F\left(\bar{F}_{n, i} ; f, g ; c_{0}, P_{c_{i}, c_{n}}\right) F\left(P_{a+1}^{\prime} ; f, g ; c_{i}\right) F\left(P_{b+1}{ }^{\prime \prime} ; f, g ; c_{n}\right)}{y^{2}} \\
& =F\left(\bar{F}_{n, i} ; f, g ; c_{0}, P_{c_{i}, c_{n}}\right) \sum_{k=1}^{a+1}(y z)^{k-1} \times \sum_{k=1}^{b+1}(y z)^{k-1} .
\end{aligned}
$$

Clearly, $\widetilde{F}_{a, b}^{i, i}$ is a trivial tree, and by Lemma 1 , we can get

$$
\sum_{T \in S\left(\mathcal{F}_{a, i} ; c_{0}\right)}=\sum_{t=1}^{l_{i}+1} y^{t} z^{t-1}+y^{l_{i}} z^{l_{i}+1} \times \sum_{t=1}^{a+1} y^{t} z^{t-1} \times \sum_{t=1}^{b+1} y^{t} z^{t-1} .
$$

Combining equations (20)-(24), we can obtain equation (18), and thus the lemma is proved.

With Lemmas 1 and 7, we can obtain the subtree generating function of generalized fan graphs.

Theorem 2. Let $F(n)=(V(F(n)), E(F(n)) ; f, g)$ be a weighted generalized fan graph, with $f(v)=y(v \in V(F(n)))$ and $g(e)=z(e \in E(F(n)))$; then,

$$
F(F(n) ; f, g)=F\left(\widetilde{F}_{0,0}^{n, 1} ; f, g ; c_{0}\right)+F\left(\widetilde{T}_{0,0}^{n, 1} ; f, g\right)
$$

with $F\left(\widetilde{F}_{a, b}^{n, i} ; f, g ; c_{0}\right)(a, b \geq 0 ; 1 \leq i \leq n)$ as in equation (18).

$$
\begin{aligned}
F\left(\widetilde{T}_{a, b}^{n, i} ; f, g\right)= & \sum_{k=1}^{b+1}(y z)^{k} \sum_{k=1}^{l_{n}+1}(y z)^{k-1}\left(F\left(\widetilde{T}_{a, m_{n-1}}^{n-1, i} ; f, g\right)-F\left(\widetilde{T}_{a, m_{n-1}-1}^{n-1, i} ; f, g\right)\right) \\
& +F\left(\widetilde{T}_{a, m_{n-1}}^{n-1, i} ; f, g\right)+\sum_{k=1}^{l_{n}+b+1}\left(l_{n}+b-k+2\right) y^{k} z^{k-1}
\end{aligned}
$$

and $\quad F\left(\widetilde{T}_{a, b}^{i, i} ; f, g\right)=\sum_{k=0}^{b-1}(b-k) y^{k+1} z^{k}+\sum_{k=0}^{a-1}(a-k)$ $y^{k+1} z^{k}+\sum_{k=0}^{l_{i}-1}\left(l_{i}^{a, b}-k\right) y^{k+1} z^{k}+\sum_{k=1}^{a+1}(y z)^{k-1}$, $\times \sum_{k=1}^{l_{i}+1} y^{k} z^{k-1} \times \sum_{k=1}^{b+1}(y z)^{k-1}(a, b \geq 0 ; 1 \leq i \leq n)$.

Next, we discuss some special cases of the generalized fan graphs.

Definition 8. Let $F\left(l_{1}, \ldots, l_{n} ; m_{1}, \ldots, m_{n-1}\right)$ be a generalized fan graph and $k$ be a positive integer; if $l_{i}=m_{j}=k(i=1, \ldots, n ; j=1, \ldots, n-1)$, then the generalized fan graph is called the $k$ regular fan graph, denoted by $F_{n, k}(n \geq 3 ; k \geq 0)$. Obviously, $F_{n, k}$ has $(2 n-1) k+n+1$ vertices and $(2 n-1)(k+1)$ edges.
From Theorem 2, the following corollaries follow as immediate consequences.

Corollary 4. Let $F_{n, k}=\left(V\left(F_{n, k}\right), E\left(F_{n, k}\right) ; f, g\right)$ be a weighted $k$ regular fan graph, with $f(v)=y\left(v \in V\left(F_{n, k}\right)\right)$ and $g(e)=z\left(e \in E\left(F_{n, k}\right)\right)$; then,

$$
F\left(F_{n, k} ; f, g\right)=F\left(F_{0,0}^{n, 1} ; f, g ; c_{0}\right)+F\left(T_{0,0}^{n, 1} ; f, g\right),
$$

with

$$
\begin{aligned}
F\left(F_{a, b}^{n, i} ; f, g ; c_{0}\right)= & \sum_{t=i}^{n-1} \frac{n-t}{y^{n-t}} \sum_{j=t / n-1\rfloor}^{k} F\left(F_{a, k-j}^{t, i} ; f, g ; c_{0}\right)(y z)^{(k+1)(n-t)+j} \times \sum_{j=1}^{b+1} y^{j} z^{j-1}\left(\sum_{j=1}^{k+1} j y^{j} z^{j-1}\right)^{n-t} \\
& +F\left(F_{a, k}^{n-1, i} ; f, g ; c_{0}\right) \sum_{j=1}^{k+b+2}(y z)^{j-1}+\frac{(n-i+1)}{y^{n-i-1}}(y z)^{(k+1)(n-i+1)}\left(\sum_{j=1}^{k+1} j y^{j} z^{j-1}\right)^{n-i} \sum_{j=1}^{a+1}(y z)^{j-1} \sum_{j=1}^{b+1}(y z)^{j-1},
\end{aligned}
$$


and $F\left(F_{a, b}^{i, i} ; f, g ; c_{0}\right)=\sum_{t=1}{ }^{k+1} y^{t} z^{t-1}+y^{k} z^{k+1} \sum_{t=1}^{a+1} y^{t} z^{t-1}$

$\sum_{t=1}^{b+1} y^{t} z^{t-1}(a, b \geq 0 ; 1 \leq i \leq n)$.

$$
\begin{aligned}
F\left(T_{a, b}^{n, i} ; f, g\right)= & \sum_{j=1}^{b+1}(y z)^{j} \sum_{j=1}^{k+1}(y z)^{j-1}\left(F\left(T_{a, k}^{n-1, i} ; f, g\right)-F\left(T_{a, k-1}^{n-1, i} ; f, g\right)\right) \\
& +F\left(T_{a, k}^{n-1, i} ; f, g\right)+\sum_{j=1}^{k+b+1}(k+b-j+2) y^{j} z^{j-1},
\end{aligned}
$$
$\underset{\left.(a-j) y^{j+1} z^{j}+\sum_{j=0}^{i, i} ; \underset{k-1}{k-1}, g\right)=\sum_{j=0}^{b-}(k-j) y^{j+1} z^{j},}{a n d} \quad 1(b-j) y^{j+1} z^{j}+\sum_{j=0}^{a-1}$ $+\sum_{j=1}^{a+1}(y z)^{j-1} \sum_{j=1}^{k+1} y^{j} z^{j-1} \sum_{j=1}^{b+1}(y z)^{j-1}(a, b \geq 0 ; 1 \leq i \leq n)$.

By substituting $y=1, z=1$ into equations (25) and (27), we have the corresponding subtree number indices.
Corollary 5. The subtree number index of the generalized fan graph $F(n)$ is

$$
\operatorname{STN}(F(n))=F(F(n) ; 1,1)=\operatorname{STN}\left(\widetilde{F}_{0,0}^{n, 1} ; c_{0}\right)+\operatorname{STN}\left(\widetilde{T}_{0,0}^{n, 1}\right),
$$

with

$$
\begin{aligned}
\operatorname{STN}\left(\widetilde{F}_{a, b}^{n, i} ; c_{0}\right)= & (b+1)\left(\sum_{t=i}^{n-1} \operatorname{STN}\left(\bar{F}_{n, t+1} ; c_{0}, P_{c_{t+1}, c_{n}}\right) \sum_{j=t t / n-1\rfloor}^{m_{t}} \operatorname{STN}\left(\widetilde{F}_{a, m_{t}-j}^{t, i} ; c_{0}\right)+(a+1) \operatorname{STN}\left(\bar{F}_{n, i} ; c_{0}, P_{c_{i}, c_{n}}\right)\right) \\
& +\operatorname{STN}\left(\widetilde{F}_{a, m_{n-1}}^{n-1, i} ; c_{0}\right)\left(l_{n}+b+2\right),
\end{aligned}
$$

$$
\begin{aligned}
& \text { and } \operatorname{STN}\left(\bar{F}_{n, i} ; c_{0}, P_{c_{i}, c_{n}}\right) \quad=\sum_{t=i}^{n}\left(\prod_{j=i}^{n}\left(l_{j}+1\right)\left(l_{j}+2\right) \quad \text { and } \quad \operatorname{STN}\left(\widetilde{T}_{a, b}^{i, i}\right)=(1 / 2)(a \quad(a+1)+b(b+1)\right. \\
& \left./ 2^{n-i}\left(l_{t}+1\right)\left(l_{t}+2\right)\right) \\
& \operatorname{STN}\left(\widetilde{F}_{a, b}, i, c_{0}\right)=(a+1)(b+1)+l_{i}+1(a, b \geq 0 ; 1 \leq i \leq n) \text {. } \\
& \left.+l_{i}\left(l_{i}+1\right)\right)+(a+1)(b+1)\left(l_{i}+1\right)(a, b \geq 0 ; 1 \leq i \leq n) \text {. }
\end{aligned}
$$$$
\operatorname{STN}\left(\widetilde{T}_{a, b}^{n, i}\right)=(b+1)\left(l_{n}+1\right)\left(\operatorname{sTN}\left(\widetilde{T}_{a, m_{n-1}}^{n-1, i}\right)-\operatorname{STN}\left(\widetilde{T}_{a, m_{n-1}-1}^{n-1, i}\right)\right)
$$$$
+\operatorname{STN}\left(\widetilde{T}_{a, m_{n-1}}^{n-1, i}\right)+\frac{1}{2}\left(l_{n}+b+1\right)\left(l_{n}+b+2\right),
$$

Corollary 6. The subtree number index of the $k$ regular fan graph $F_{n, k}$ is

$$
\operatorname{STN}\left(F_{n, k}\right)=F\left(F_{n, k} ; 1,1\right)=\operatorname{STN}\left(F_{0,0}^{n, 1} ; c_{0}\right)+\operatorname{STN}\left(T_{0,0}^{n, 1}\right),
$$

with

$$
\begin{aligned}
\operatorname{STN}\left(F_{a, b}^{n, i} ; c_{0}\right)= & (b+1)\left(\sum_{t=i}^{n-1}(n-t) \sum_{j=t / n-1\rfloor}^{k} \operatorname{STN}\left(F_{a, k-j}^{t, i} ; c_{0}\right) \frac{\left(k^{2}+3 k+2\right)^{n-t}}{2^{n-t}}+(a+1)(n-i+1) \frac{\left(k^{2}+3 k+2\right)^{n-i}}{2^{n-i}}\right) \\
& +\operatorname{STN}\left(F_{a, k}^{n-1, i} ; c_{0}\right)(k+b+2),
\end{aligned}
$$

and $\operatorname{STN}\left(\widetilde{F}_{a, b}^{i, i} ; c_{0}\right)=(a+1)(b+1)+k+1(a, b \geq 0 ; 1 \leq i \leq$ $n)$.

$$
\begin{aligned}
\operatorname{STN}\left(T_{a, b}^{n, i}\right)= & (b+1)(k+1)\left(\operatorname{STN}\left(T_{a, k}^{n-1, i}\right)-\operatorname{STN}\left(T_{a, k-1}^{n-1, i}\right)\right) \\
& +\operatorname{STN}\left(T_{a, k}^{n-1, i}\right)+\frac{1}{2}(k+b+1)(k+b+2),
\end{aligned}
$$

and $\operatorname{STN}\left(T_{a, b}^{i, i}\right)=(1 / 2)(a(a+1)+b(b+1)+k(k+1))+$ $(a+1)(b+1)(k+1)(a, b \geq 0 ; 1 \leq i \leq n)$.

By Corollary 6, we can get the number of subtrees in $F_{n, k}(n=3,4, \ldots, 12 ; k=0,1, \ldots, 4)$ as shown in Table 2.

3.3. Subtree Generating Functions of Unicyclic Graphs. In order to solve the subtree generating function of the 
TABLE 2: The subtree number index $\operatorname{STN}\left(F_{n, k}\right)$ of $F_{n, k}(n=3, \ldots, 12 ; k=0, \ldots, 4)$.

\begin{tabular}{|c|c|c|c|c|c|}
\hline$n$ & $\operatorname{STN}\left(F_{n, 0}\right)$ & $\operatorname{STN}\left(F_{n, 1}\right)$ & $\operatorname{STN}\left(F_{n, 2}\right)$ & $\operatorname{STN}\left(F_{n, 3}\right)$ & $\operatorname{STN}\left(F_{n, 4}\right)$ \\
\hline 3 & 25 & 237 & 942 & 2590 & 5775 \\
\hline 4 & 70 & 1878 & 14889 & 68434 & 229620 \\
\hline 5 & 204 & 16416 & 258651 & 1963014 & 9793890 \\
\hline 6 & 616 & 148251 & 4583355 & 57021154 & 421194660 \\
\hline 7 & 1901 & 1350654 & 81524559 & 1659516014 & 18133098555 \\
\hline 8 & 5932 & 12331656 & 1451078262 & 48312035578 & 780774946200 \\
\hline 9 & 18605 & 112645497 & 25831232655 & 1406527479494 & 33619284887595 \\
\hline 10 & 58480 & 1029092802 & 459841504026 & 40949040340498 & 1447611875610240 \\
\hline 11 & 183982 & 9401689836 & 8186017062132 & 1192173807684958 & 62332696770629760 \\
\hline 12 & 579027 & 85893370743 & 145726106717904 & 34708469794621098 & 2683982668276977405 \\
\hline
\end{tabular}

generalized wheel graphs, we first study the subtree generating function of the unicyclic graphs.

Lemma 8 (see [14]). Let $T$ be a weighted tree with more than 2 vertices, $u$ and $v$ be its two distinct vertices, and $P_{u v}$ be a path of $T$ connecting $u$ and $v$ with length $l$, vertex set $V\left(P_{u v}\right)=\left\{x_{i} \mid i=1,2, \ldots, l-1\right\} \cup\{u, v\}$, and $E\left(P_{u v}\right)=\left(u, x_{1}\right) \cup\left\{\left(x_{i}, x_{i+1}\right) \mid i=1,2, \ldots, l-2\right\} \cup\left(x_{l-1}, v\right)$; moreover, denote by $T_{u}, T_{v}, T_{x_{i}}(i=1,2, \ldots, l-1)$ the weighted tree of $T \backslash E\left(P_{u v}\right)$ that contains $u, v, x_{i}$, respectively. Then,

$$
F(T ; f, g ; u, v)=f(u) f(v) \prod_{i=1}^{l-1} f\left(x_{i}\right) \prod_{e \in E\left(P_{u v}\right)} g(e),
$$

where $f(\widetilde{v})=F\left(T_{\widetilde{v}} ; f, g ; \widetilde{v}\right)\left(\widetilde{v} \in\left\{u, v, x_{i}(i=1, \ldots, l-1)\right\}\right)$.

Theorem 3. Let $U_{n}=\left(V\left(U_{n}\right), E\left(U_{n}\right) ; f, g\right)$ be a weighted unicyclic graph with girth $n$, vertex set $V\left(U_{n}\right)=\left\{v_{1}, v_{2}, \ldots\right.$, $\left.v_{n}\right\}$, vertex weight function $f(v)=y\left(v \in V\left(U_{n}\right)\right.$, and edge weight function $g(e)=z\left(e \in E\left(U_{n}\right)\right)$, having trees $T_{i}(i=$ $1,2, \ldots, n)$ attached to vertex $v_{i}$ on the cycle; then,

$$
\begin{aligned}
F\left(U_{n} ; f, g\right)= & \sum_{i=1}^{n-1} \sum_{j=1}^{n} \prod_{k=j}^{j+i} F\left(T_{k} ; f, g ; v_{k}\right) \prod_{e \in E\left(P_{v_{j} v_{j+i}}\right)} g(e) \\
& +\sum_{i=1}^{n} F\left(T_{i} ; f, g\right),
\end{aligned}
$$

where $P_{v_{j} v_{j+i}}=v_{j} v_{j+1} v_{j+2} \ldots v_{j+i}$ is the path connecting $v_{j}$ and $v_{j+i}$; here we specify that $T_{i+n}=T_{i}, v_{i+n}=v_{i}$.

Proof. The theorem holds through dividing the subtrees of $U_{n}$ into the following three categories:
(1) Containing no vertex and no edge on the cycle.

(2) Containing only one vertex and no edge on the cycle.

(3) Containing only $i+1$ vertices and $i$ edges $(i=1, \ldots, n-1)$ on the cycle.

3.4. Subtree Generating Function of the Generalized Wheel Graphs. Next, we consider the subtree generating function of the generalized wheel graph $W(n)$.

Definition 9. Let $\bar{F}_{p, q}^{n}(p, q \in\{1,2, \ldots, n\})$ be the generalized fan graph of $W(n)\left(e_{p-1, m_{p-1}+1}^{*} \cup e_{q, 1}^{*} \cup \cup_{i=q+1}^{p-1} \widetilde{e}_{i, 1}\right)$ that contains $c_{0}, c_{p}, c_{q}$, and denote by $P_{c_{p}, c_{q}}^{\prime}$ the path tree of $\bar{F}_{p, q}^{n} \backslash\left(\cup_{i=p}^{q} \widetilde{e}_{i, l_{i}+1}\right)$ that contains $c_{p}, c_{q}$.

With Lemma 6, the following lemma follows immediately.

Lemma 9. Keeping above symbols and definitions, we have $F\left(\bar{F}_{p, q}^{n} ; f, g ; c_{0}, P_{c_{p}, c_{q}}^{\prime}\right)=\sum_{i=p}^{x} \frac{(y z)^{l_{i}+1+\sum_{k=p}^{x-1}\left(m_{k}+1\right)}}{y^{x-p-1} \sum_{j=1}^{l_{i}+1} j y^{j} z^{j-1}} \prod_{j=p}^{x} \sum_{k=1}^{l_{j}+1} k y^{k} z^{k-1}$,

with

$$
x= \begin{cases}q, & \text { if } q \geq p \\ n+q, & \text { otherwise }\end{cases}
$$

Theorem 4. Let $W(n)=(V(W(n)), E(W(n)) ; f, g)$ be a weighted generalized wheel graph with vertex weight function $f(v)=y(v \in V(W(n)))$ and edge weight function $g(e)=$ $z(e \in E(W(n)))$ (see Definition 4); then, 


$$
\begin{aligned}
F(W(n) ; f, g)= & \sum_{p=1}^{n} \sum_{k \neq f|p| n]}^{m_{p}}\left(\begin{array}{c}
\frac{1}{y} \sum_{i=1}^{p-1} \sum_{j=0}^{m_{i}}(y z)^{k+j} F\left(\bar{F}_{p+1, i}^{n} ; f, g ; c_{0}, P_{c_{p+1}, c_{i}}\right) \times F\left(\widetilde{F}_{m_{i}-j, m_{p}-k}^{p, i+1} ; f, g ; c_{0}\right) \\
+F\left(\bar{F}_{p+1, p}^{n} ; f, g ; c_{0}, P_{c_{p+1}, c_{p}}\right) \sum_{i=0}^{m_{p}-k}(y z)^{k+i}
\end{array}\right) \\
& +\sum_{i=1}^{n} \sum_{j=i / n]}^{m_{i}}(y z)^{j}\left(\sum_{k=0}^{m_{1}-j}(y z)^{k}\right)^{1-[i-1 / n]}\left(\left(F\left(\widetilde{T}_{0, m_{i}-j}^{i, 1} ; f, g\right)-F\left(\widetilde{T}_{m_{1}, m_{i}-j}^{i, 2} ; f, g\right)-\sum_{k=0}^{l_{1}-1}\left(l_{1}-k\right) y^{k+1} z^{k}\right)\right)^{[i-1 / n]} \\
& \prod_{j=i+f i-1 / n]}^{n} \sum_{t=1}^{l_{j}+1} y^{t} z^{t-1} \prod_{j=i+1}^{n} y^{m_{j}} z^{m_{j}+1}+F\left(\widetilde{T}_{0, m_{n}}^{n, 1} ; f, g\right)+F\left(\widetilde{F}_{0, m_{n}}^{n, 1} ; f, g ; c_{0}\right),
\end{aligned}
$$

with $F\left(\widetilde{T}_{a, b}^{n, i} ; f, g ; c_{0}\right), F\left(\widetilde{T}_{a, b}^{n, i} ; f, g\right), F\left(\bar{F}_{p, q}^{n} ; f, g ; c_{0}, P_{c_{p}, c_{q}}\right)$ as in equations (18), (26), and (38), respectively, and $\bar{F}_{i+n, q}^{n}=\bar{F}_{i, q}^{n}(1,2, \ldots, n)$.

Proof. We first divide the subtrees of generalized wheel graph into two categories:
(1) Not containing the center vertex $c_{0}$.

(2) Containing the center vertex $c_{0}$.

Obviously, $W(n) \backslash c_{0}$ is a unicyclic graph; with Theorem 3 , we have the subtree generating function of case (1) as

$$
\begin{aligned}
F\left(W(n) \backslash c_{0} ; f, g\right)=\sum_{i=1}^{n} \sum_{j=i / n\rfloor}^{m_{i}}(y z)^{j} & \left(\left(F\left(\widetilde{T}_{0, m_{i}-j}^{i, 1} ; f, g\right)-F\left(\widetilde{T}_{m_{1}, m_{i}-j}^{i, 2} ; f, g\right)-\sum_{k=0}^{l_{1}-1}\left(l_{1}-k\right) y^{k+1} z^{k}\right)\right) \\
& \left(\sum_{k=0}^{m_{1}-j}(y z)^{k}\right)^{1-[i-1 / n]} \prod_{j=i+1}^{n} y^{m_{j}} z^{m_{j}+1} \prod_{j=i+f i-1 / n]}^{n} \sum_{t=1}^{l_{j}+1} y^{t} z^{t-1}+F\left(\widetilde{T}_{0, m_{n}}^{n, 1} ; f, g\right) .
\end{aligned}
$$

We further partition the subtree of case (2) into four categories.

$$
S\left(W(n) ; c_{0}\right)=S_{1} \cup S_{2} \cup S_{3} \cup S_{4},
$$

where

(i) $S_{1}$ is the set of subtrees in $S\left(W(n) ; c_{0}\right)$ that does not contain edge $e_{n, m_{n}+1}^{*}$.

(ii) $S_{2}$ is the set of subtrees in $S\left(W(n) ; c_{0}\right)$ that contains edge set $\cup_{j=m_{n}+1-i}^{m_{n}+1} e_{n, j}^{*}$, but not $e_{n, m_{n}-i}^{*}(i=0,1, \ldots$, $\left.m_{n}-1\right)$.

(iii) $S_{3}$ is the set of subtrees in $S\left(W\left(\begin{array}{c}n \\ m\end{array} ; c_{0}\right)\right.$ that contains edge set $\cup_{i=n-p+1}^{n} \cup \cup_{j=1}^{m_{i}+1} e_{i, j}^{*} \cup \cup_{j=m_{n-p}+2-q}^{m_{n-p} e_{n-p, j}^{*}}$, but not $e_{n-p, m_{n-p}-q+1}^{*}(p=1,2, \ldots, n-2 ; q=0,1, \ldots$, $\left.m_{n-p}\right)$.

(iv) $S_{4}$ is the set of subtrees in $S\left(W(n) ; c_{0}\right)$ that contains edge set $\cup_{i=2}^{n} \cup \cup_{j=1}^{m_{i}+1} e_{i, j}^{*} \cup \cup_{j=m_{1}+2-q}^{m_{1}+1} e_{1, j}^{*}$, but not $e_{1, m_{1}-q+1}^{*}\left(q=0,1, \ldots, m_{1}\right)$.
With equation (18), we have the subtree generating function of case $S_{1}$ as

$$
\sum_{T_{1} \in S_{1}} w\left(T_{1}\right)=F\left(\widetilde{F}_{0, m_{n}}^{n, 1} ; f, g ; c_{0}\right) .
$$

We further categorize the subtrees in set $S_{2}$ into two cases:

$$
S_{2}=S_{2,1} \cup S_{2,2}
$$

(v) $S_{2,1}$ is the set of subtrees in $S_{2}$ that contains edge set $\left(\cup_{i=1}^{p-1} \cup \cup_{j=1}^{m_{i}+1} e_{i, j}^{*}\right) \cup \cup_{j=1}^{q-1} e_{p, j}^{*}(p=1,2, \ldots, n-1 ; q=$ $\left.1,2, \ldots, m_{p}+1\right)$, but not $e_{p, q}^{*}$.

(vi) $S_{2,2}$ is the set of subtrees in $S_{2}$ that contains edge set $\cup_{i=1}^{n-1} \cup_{j=1}^{m_{i}+1} e_{i, j}^{*}$.

Then, we have 
TABLE 3: The subtree number index $\operatorname{STN}\left(W_{n, k}\right)$ of the $k$ regular wheel graphs $W_{n, k}(n=3, \ldots, 10 ; k=0,1, \ldots, 4)$.

\begin{tabular}{lccccc}
\hline$n$ & $\operatorname{STN}\left(W_{n, 0}\right)$ & $\operatorname{STN}\left(W_{n, 1}\right)$ & $\operatorname{STN}\left(W_{n, 2}\right)$ & $\operatorname{STN}\left(W_{n, 3}\right)$ & $\mathrm{STN}\left(W_{n, 4}\right)$ \\
\hline 3 & 38 & 854 & 6012 & 25508 & 80810 \\
4 & 112 & 7221 & 101139 & 713728 & 3388080 \\
5 & 332 & 64245 & 1784919 & 20793884 & 146828850 \\
6 & 1007 & 582668 & 31779252 & 607320008 & 6352788995 \\
7 & 3110 & 5314153 & 566171601 & 17710247828 & 274109511640 \\
8 & 9704 & 48533965 & 10083174375 & 515947702112 & 11812025781160 \\
9 & 30431 & 443386514 & 179530660440 & 15024717676460 & 508755146972555 \\
10 & 95643 & 4050782145 & 3196186084833 & 437460940097784 & 21908623666992075 \\
\hline
\end{tabular}

$$
\begin{aligned}
\sum_{T_{2} \in S_{2}} w\left(T_{2}\right)= & \sum_{p=1}^{m_{n}} \prod_{j=1}^{p} f\left(v_{n, m_{n}-j+1}^{*}\right) g\left(e_{n, m_{n}-j+2}^{*}\right)\left(\sum_{T_{2,1} \in S_{2,1}} w\left(T_{2,1}\right)+\sum_{T_{2,2} \in S_{2,2}} w\left(T_{2,2}\right)\right) \\
= & \sum_{p=1}^{m_{n}} \frac{(y z)^{p}}{y} \sum_{j=1}^{n-1} F\left(\bar{F}_{1, j}^{n} ; f, g ; c_{0}, P_{c_{1}, c_{j}}^{\prime}\right) \sum_{i=0}^{m_{j}} F\left(\widetilde{F}_{m_{j}-i, m_{n}-p}^{n, j+1} ; f, g ; c_{0}\right) \prod_{s=1}^{i} f\left(v_{j, s}^{*}\right) g\left(e_{j, s}^{*}\right) \\
& +F\left(\bar{F}_{1, n}^{n} ; f, g ; c_{0}, P_{c_{1}, c_{n}}^{\prime}\right) \times F\left(P_{m_{n}+1-p}^{\prime \prime} ; f, g ; c_{n}\right) \\
= & \sum_{p=1}^{m_{n}} y^{p-1} z^{p}\left(\sum_{j=1}^{n-1} F\left(\bar{F}_{1, j}^{n} ; f, g ; c_{0}, P_{c_{1}, c_{j}}^{\prime}\right) \sum_{i=0}^{m_{j}}(y z)^{i} F\left(\widetilde{F}_{m_{j}-i, m_{n}-p}^{n, j+1} ; f, g ; c_{0}\right)+F\left(\bar{F}_{1, n}^{n} ; f, g ; c_{0}, P_{c_{1}, c_{n}}^{\prime}\right) \sum_{q=1}^{m_{n}+1-p} y^{q} z^{q-1}\right) .
\end{aligned}
$$

Similar to the analysis of case $S_{2}$, we have

$$
\begin{aligned}
& \sum_{T_{3} \in S_{3}} w\left(T_{3}\right)=\frac{1}{y} \sum_{p=2}^{n-1} \sum_{k=0}^{m_{p}} \prod_{j_{1}=1}^{k} f\left(v_{p, m_{p}-j_{1}+1}^{*}\right) g\left(e_{p, m_{p}-j_{1}+2}^{*}\right)\left(\begin{array}{c}
\sum_{i=1}^{p-1} F\left(\bar{F}_{p+1, i}^{n} ; f, g ; c_{0}, P_{c_{p+1}, c_{i}}^{\prime}\right) \sum_{j=0}^{m_{i}} F\left(\widetilde{F}_{m_{i}-j, m_{p}-k}^{p, i+1} ; f, g ; c_{0}\right) \prod_{s=1}^{j} f\left(v_{i, s}^{*}\right) g\left(e_{i, s}^{*}\right) \\
+F\left(\bar{F}_{p+1, p}^{n} ; f, g ; c_{0}, P_{c_{p+1}, c_{p}}^{\prime}\right) F\left(P_{m_{p}+1-k}^{\prime \prime} ; f, g ; c_{p}\right)
\end{array}\right) \\
& =\sum_{p=2}^{n-1} \sum_{k=0}^{m_{p}} y^{k-1} z^{k}\left(\sum_{i=1}^{p-1} F\left(\bar{F}_{p+1, i}^{n} ; f, g ; c_{0}, P_{c_{p+1}, c_{i}}\right) \sum_{j=0}^{m_{i}} F\left(\widetilde{F}_{m_{i}-j, m_{p}-k}^{p, i+1} ; f, g ; c_{0}\right)(y z)^{j}\right)+F\left(\bar{F}_{p+1, p}^{n} ; f, g ; c_{0}, P_{c_{p+1}, c_{p}}\right)^{m_{p}+1-k} \sum_{q=1}^{y^{q}} y^{q} z^{q-1}, \\
& \sum_{T_{4} \in S_{4}} w\left(T_{4}\right)=\frac{1}{y} F\left(\bar{F}_{2,1}^{n} ; f, g ; c_{0}, P_{c_{2}, c_{1}}^{\prime}\right) \sum_{p=0}^{m_{1}} \prod_{i=1}^{p} f\left(v_{1, m_{1}-i+1}^{*}\right) g\left(v_{1, m_{1}-i+2}^{*}\right) F\left(P_{m_{1}+1-p}^{\prime \prime} ; f, g ; c_{1}\right) \\
& =F\left(\bar{F}_{2,1}^{n} ; f, g ; c_{0}, P_{c_{2}, c_{1}}\right) \sum_{p=0}^{m_{1}} y^{p-1} z^{p} \times \sum_{j=1}^{m_{1}+1-p} y^{j} z^{j-1}
\end{aligned}
$$

Equation (40) follows from equations (41) to (47).

With Theorem 4, we can also obtain the subtree generating functions of some special generalized wheel graphs.

Definition 10. Let $W\left(l_{1}, \ldots, l_{n} ; m_{1}, \ldots, m_{n}\right)$ be a generalized wheel graph and $k$ be a positive integer; the generalized wheel graph is called $k$ regular wheel graph, denoted by $W_{n, k}(n \geq 3 ; k \geq 0)$, if $l_{i}=m_{i}=k(i=1, \ldots, n)$. Clearly, $W_{n, k}$ has $n(2 k+1)+1$ vertices and $2 n(k+1)$ edges.

Corollary 7. Let $W_{n, k}=\left(V\left(W_{n, k}\right), E\left(W_{n, k}\right) ; f, g\right)$ be a weighted $k$ regular wheel graph, with $f(v)=y\left(v \in V\left(W_{n, k}\right)\right)$ and $g(e)=z\left(e \in E\left(W_{n, k}\right)\right)$; then, 


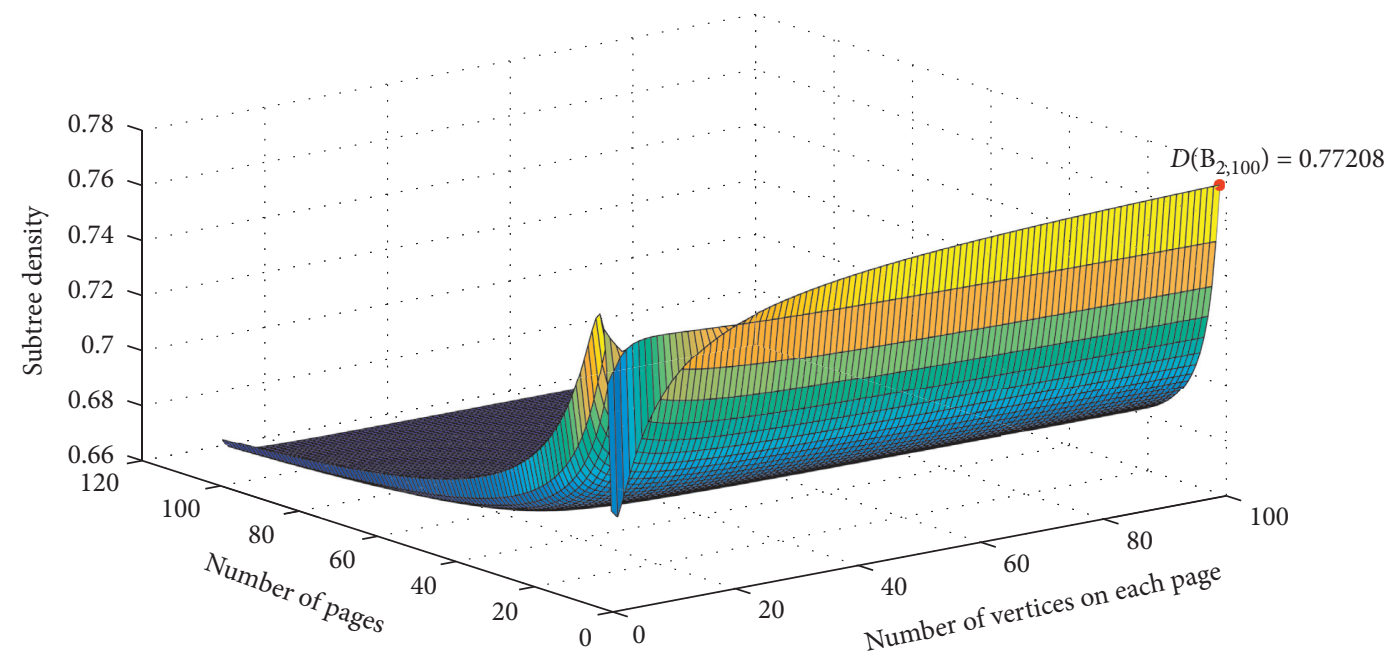

FIgURE 2: Subtree densities of $B_{n, k}(n=2,3, \ldots, 101 ; k=1,2, \ldots, 100)$.

TABLe 4: Related data for $B_{n, k}(n=2,3, \ldots, 11 ; k=1,2,3)$.

\begin{tabular}{|c|c|c|c|c|c|c|}
\hline \multirow{2}{*}{$n$} & \multicolumn{2}{|c|}{$k=1$} & \multicolumn{2}{|c|}{$k=2$} & \multicolumn{2}{|c|}{$k=3$} \\
\hline & $P_{y}\left(B_{n, 1}\right)$ & $D\left(B_{n, 1}\right)$ & $P_{y}\left(B_{n, 2}\right)$ & $D\left(B_{n, 2}\right)$ & $P_{y}\left(B_{n, 3}\right)$ & $D\left(B_{n, 3}\right)$ \\
\hline$n=2$ & 160 & 0.695652 & 948 & 0.692982 & 3434 & 0.699959 \\
\hline$n=3$ & 640 & 0.740741 & 7738 & 0.745472 & 47624 & 0.749937 \\
\hline$n=4$ & 2524 & 0.760699 & 63416 & 0.757007 & 667458 & 0.753912 \\
\hline$n=5$ & 9752 & 0.765704 & 507894 & 0.753722 & 9081980 & 0.746593 \\
\hline$n=6$ & 36880 & 0.763656 & 3954124 & 0.746654 & 119376902 & 0.738012 \\
\hline$n=7$ & 136704 & 0.758792 & 29977970 & 0.739331 & 1521704016 & 0.730391 \\
\hline$n=8$ & 497716 & 0.753086 & 222103008 & 0.732726 & 18907602266 & 0.724007 \\
\hline$n=9$ & 1783768 & 0.747392 & 1613730478 & 0.727007 & 230033554532 & 0.718704 \\
\hline$n=10$ & 6305512 & 0.742052 & 11533135796 & 0.722104 & 2750146800750 & 0.714269 \\
\hline$n=11$ & 22023584 & 0.737177 & 81280358058 & 0.717896 & 32400637534328 & 0.71052 \\
\hline
\end{tabular}

$P_{y}\left(B_{n, k}\right)$ stands for $\left.\left(\partial F\left(B_{n, k} ; y, 1\right) / \partial y\right)\right|_{y=1}(n=2,3, \ldots, 11 ; k=1,2,3)$.

$$
\begin{aligned}
& F\left(W_{n, k} ; f, g\right)=F\left(F_{0, k}^{n, 1} ; f, g ; c_{0}\right)+\left(y^{k} z^{k+1}\right)^{n-1} \sum_{i=0}^{k} \sum_{j=0}^{k-i}(y z)^{i+j}\left(\sum_{j=1}^{k+1} y^{j} z^{j-1}\right)^{n} \\
& +\sum_{p=1}^{n} \sum_{t=\lfloor p / n\rfloor}^{k}\left(\begin{array}{c}
\sum_{i=1}^{p-1} \frac{(n-p+i)}{y^{n-p+i-1}} \sum_{j=0}^{k} F\left(F_{k-j, k-t}^{p, i+1} ; f, g ; c_{0}\right)(y z)^{(k+1)(n-p+i)+t+j} \times\left(\sum_{s=1}^{k+1} s y^{s} z^{s-1}\right)^{n-p+i-1} \\
+\frac{n(y z)^{n(k+1)}}{y^{n-2}}\left(\sum_{s=1}^{k+1} s y^{s} z^{s-1}\right)^{n-1} \sum_{i=0}^{k-t}(y z)^{t+i}
\end{array}\right) \\
& +\sum_{i=2}^{n} \sum_{j=\lfloor i / n\rfloor}^{k}(y z)^{j}\left(F\left(T_{0, k-j}^{i, 1} ; f, g\right)-F\left(T_{k, k-j}^{i, 2} ; f, g\right)-\sum_{t=0}^{k-1}(k-t) y^{t+1} z^{t}\right) \times\left(y^{k} z^{k+1} \sum_{t=1}^{k+1} y^{t} z^{t-1}\right)^{n-i} \\
& +F\left(T_{0, k}^{n, 1} ; f, g\right),
\end{aligned}
$$

with $F\left(F_{a, b}^{n, i} ; f, g ; c_{0}\right)$ and $F\left(T_{a, b}^{n, i} ; f, g\right)$ as in equations (28) and (29), respectively.
Substituting $y=1, z=1$ into equations (40) and (48), we have the following corollaries. 
Corollary 8. The subtree number index of generalized wheel graph $W(n)$ is

$$
\begin{aligned}
\operatorname{STN}(W(n))= & \sum_{p=1}^{n} \sum_{k=1 p / n\rfloor}^{m_{p}}\left(\sum_{i=1}^{p-1} \sum_{j=0}^{m_{i}} \operatorname{STN}\left(\bar{F}_{p+1, i}^{n} ; c_{0}, P_{c_{p+1}, c_{i}}^{\prime}\right) \operatorname{STN}\left(\widetilde{F}_{m_{i}-j, m_{p}-k}^{p, i+1} ; c_{0}\right)+\operatorname{STN}\left(\bar{F}_{p+1, p}^{n} ; c_{0}, P_{c_{p+1}, c_{p}}^{\prime}\right)\left(m_{p}-k+1\right)\right) \\
& +\operatorname{STN}\left(\widetilde{T}_{0, m_{n}}^{n, 1}\right)+\operatorname{STN}\left(\widetilde{F}_{0, m_{n}}^{n, 1} ; c_{0}\right)+\sum_{i=1}^{n} \sum_{j=\{i / n\rfloor}^{m_{i}}\left(\operatorname{STN}\left(\widetilde{T}_{0, m_{i}-j}^{i, 1}\right)-\operatorname{STN}\left(\widetilde{T}_{m_{1}, m_{i}-j}^{i, 2}\right)-\frac{l_{1}}{2}\left(l_{1}+1\right)\right) \\
& \times\left(m_{1}-j+1\right)^{1-\lceil i-1 / n\rceil} \prod_{j=i+\lceil i-1 / n\rceil}^{n}\left(l_{j}+1\right),
\end{aligned}
$$

with $\operatorname{STN}\left(\widetilde{F}_{a, b}^{n, i} ; c_{0}\right), \operatorname{STN}\left(\widetilde{T}_{n}^{n, i}\right)$ as in equations (31) and (32), respectively, and $\operatorname{STN}\left(\bar{F}_{p+1, i}^{n a, b} ; c_{0}, P_{c_{p+1}, c_{i}}\right)=F\left(\bar{F}_{p+1, i}^{n} ; 1,1 ; c_{0}\right.$, $\left.P_{c_{p+1}, c_{i}}^{\prime}\right)$ by substituting $y=z=1$ into equation (38).
Corollary 9. The subtree number index of the $k$ regular wheel graph $W_{n, k}$ is

$$
\begin{aligned}
\operatorname{STN}\left(W_{n, k} ; f, g\right)= & \operatorname{STN}\left(T_{0, k}^{n, 1}\right)+\sum_{p=1}^{n} \sum_{t=\{p / n\rfloor}^{k}\left(\sum_{i=1}^{p-1}(n-p+i) \sum_{j=0}^{k} \operatorname{STN}\left(F_{k-j, k-t}^{p, i+1} ; c_{0}\right) \frac{\left(k^{2}+3 k+2\right)^{n-p+i-1}}{2^{n-p+i-1}}+\frac{n}{2^{n-1}}(k+1-t)\left(k^{2}+3 k+2\right)^{n-1}\right) \\
& +\sum_{i=2}^{n} \sum_{j=\{i / n\rfloor}^{k}\left(\operatorname{STN}\left(T_{0, k-j}^{i, 1}\right)-\operatorname{STN}\left(T_{k, k-j}^{i, 2}\right)-\frac{k}{2}(k+1)\right)(k+1)^{n-i}+\operatorname{STN}\left(F_{0, k}^{n, 1} ; c_{0}\right)+\frac{(k+2)}{2}(k+1)^{n+1},
\end{aligned}
$$

with $\operatorname{STN}\left(\widetilde{F}_{a, b}^{n, i} ; c_{0}\right)$ and $\operatorname{STN}\left(\widetilde{F}_{a, b}^{n, i}\right)$ as in equations (34) and (35), respectively.

With Corollary 9, we can get the subtree number index of the $k$ regular wheel graph $W_{n, k}(n=3, \ldots, 10 ; k=0$, $\ldots, 4)$ as shown in Table 3.

\section{Subtree Density for Graphs}

The subtree generating function can be used to reveal more structure information of the graph than just subtree numbers. Next, we study the asymptotic characteristics of the subtree density of three types of graphs, namely, $k$ regular book graph $B_{n, k}, k$ regular fan graph $F_{n, k}$, and $k$ regular wheel graph $W_{n, k}$. The formal definition of the subtree density is given below.

Definition 11 (see [12]). Assume $G$ is a graph with $n$ vertices and $k$ subtrees of order $n_{1}, n_{2}, \ldots, n_{k}$. Then, $u(G)=(1 / k) \sum_{i=1}^{k} n_{i}$ denotes the average order of subtrees of $G$, and the subtree density of $G$ is defined as $D(G)=(u(G) / n)$.

By Definition 11, the subtree density of $G^{*}$ is simply

$$
D\left(G^{*}\right)=\frac{\left.\left(\partial F\left(G^{*} ; y, 1\right) / \partial y\right)\right|_{y=1}}{F\left(G^{*} ; 1,1\right) \times n\left(G^{*}\right)},
$$

where $F\left(G^{*} ; y, 1\right)$ represents the vertex generating function of $G^{*}, F\left(G^{*} ; 1,1\right)$ represents the number of subtrees of $G^{*}$, and $n\left(G^{*}\right)$ represents the number of vertices of $G^{*}$.

4.1. Subtree Density of the $k$ Regular Book Graph. According to definition of $k$ regular book graph, it is easy to know that the number of vertices in $B_{n, k}$ is

$$
n\left(B_{n, 2}\right)=n k+2 \text {. }
$$

With Corollary 1, we obtain the vertex generating function of $B_{n, k}$ :

$$
\begin{aligned}
F\left(B_{n, k} ; f, 1\right)= & (n+1) \sum_{i=0}^{k-1}(k-i) y^{i+1} \\
& +\frac{2}{y^{n}}\left(\sum_{i=1}^{k+1} y^{i}\right)^{n+1}+\frac{n+1}{\left(y^{k+2}\right)^{n-1}}\left(\sum_{i=1}^{k+1} i y^{i+k+1}\right)^{n} .
\end{aligned}
$$


TABLE 5: Related data for $F_{n, k}(n=3,4, \ldots, 15 ; k=0,1,2)$.

\begin{tabular}{|c|c|c|c|c|c|c|}
\hline \multirow{2}{*}{$n$} & \multicolumn{2}{|c|}{$k=0$} & \multicolumn{2}{|c|}{$k=1$} & \multicolumn{2}{|c|}{$k=2$} \\
\hline & $P_{y}\left(F_{n, 0}\right)$ & $D\left(F_{n, 0}\right)$ & $P_{y}\left(F_{n, 1}\right)$ & $D\left(F_{n, 1}\right)$ & $P_{y}\left(F_{n, 2}\right)$ & $D\left(F_{n, 2}\right)$ \\
\hline$n=3$ & 70 & 0.7 & 1469 & 0.688701 & 9230 & 0.699879 \\
\hline$n=4$ & 258 & 0.737143 & 16804 & 0.745651 & 213112 & 0.753336 \\
\hline$n=5$ & 937 & 0.765523 & 188646 & 0.766106 & 4770342 & 0.768465 \\
\hline$n=6$ & 3375 & 0.782699 & 2064131 & 0.773512 & 102963491 & 0.774643 \\
\hline$n=7$ & 12041 & 0.791754 & 22039684 & 0.777037 & 2157456112 & 0.778349 \\
\hline$n=8$ & 42510 & 0.796246 & 230629962 & 0.779261 & 44198270871 & 0.780998 \\
\hline$n=9$ & 148559 & 0.798490 & 2375047265 & 0.780899 & 889968667136 & 0.783027 \\
\hline$n=10$ & 514425 & 0.799691 & 24148356952 & 0.782189 & 17679691108012 & 0.784641 \\
\hline$n=11$ & 1767141 & 0.800414 & 243005062626 & 0.783241 & 347426791890924 & 0.785954 \\
\hline$n=12$ & 6028731 & 0.80091 & 2424615958415 & 0.784117 & 6766882927525253 & 0.787045 \\
\hline
\end{tabular}

$P_{y}\left(F_{n, k}\right)$ stands for $\left.\left(\partial F\left(F_{n, k} ; y,\right) / \partial y\right)\right|_{y=1}(n=3,4, \ldots, 12 ; k=0,1,2)$.

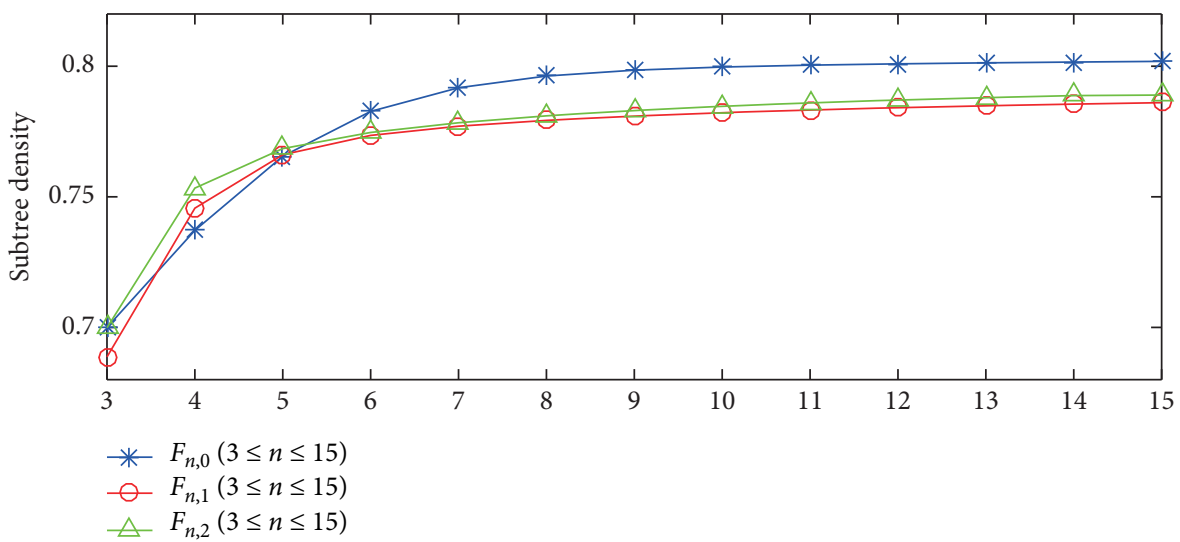

(a)

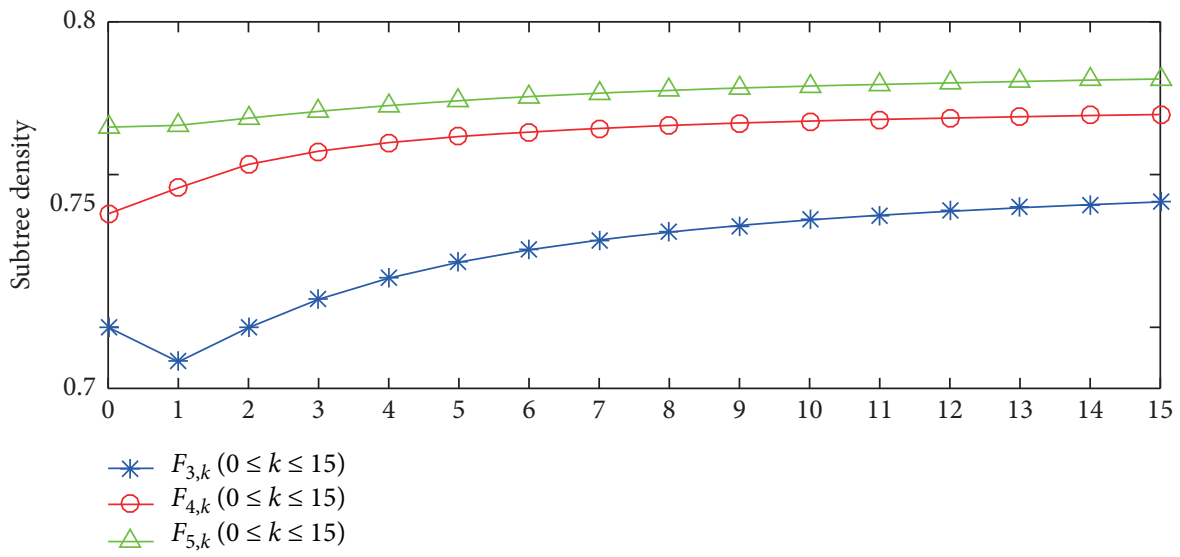

(b)

Figure 3: Subtree densities of $F_{n, k}(n=3,4, \ldots, 15 ; k=0,1, \ldots, 15)$. (a) Subtree densities of $F_{n, k}(n=3,4, \ldots, 15 ; k=0,1,2)$. (b) Subtree densities of $F_{n, k}(n=3,4, \ldots, 15 ; k=0,1, \ldots, 15)$.

Together with equations (16) and (51), we can obtain the subtree densities of $B_{n, k}$ as plotted in Figure 2, and the related data are listed in Table 4.

Observing Table 4 and Figure 2, we see that the $B_{2,100}$ has the maximal subtree density among $B_{n, k}(n=2,3, \ldots, 101 ; k=1,2, \ldots, 100)$. On the whole, it appears that the subtree density of $B_{n, k}(n=2,3, \ldots, 101 ; k=$ $1,2, \ldots, 100)$ decreases with the increase of $n$ and $k$.
4.2. Subtree Density of the $k$ Regular Fan Graph. Similarly, with Corollary 4 , we can obtain the vertex generation function of $F_{n, k}$, and combining the subtree density definition (see equation (51)), we can obtain the subtree densities of $F_{n, k}$ as shown in Table 5 and Figures 3(a) and 3(b).

From Figure 3(a), when $k \in\{0,1,2\}$ is fixed, we can observe that the subtree density of $F_{n, k}(3 \leq n \leq 15)$ gradually 
TABLE 6: Related data for $W_{n, k}(n=3,4, \ldots, 10 ; k=0,1,2)$.

\begin{tabular}{|c|c|c|c|c|c|c|}
\hline \multirow{2}{*}{$n$} & \multicolumn{2}{|c|}{$k=0$} & \multicolumn{2}{|c|}{$k=1$} & \multicolumn{2}{|c|}{$k=2$} \\
\hline & $P_{y}\left(W_{n, 0}\right)$ & $D\left(W_{n, 0}\right)$ & $P_{y}\left(W_{n, 1}\right)$ & $D\left(W_{n, 1}\right)$ & $P_{y}\left(W_{n, 2}\right)$ & $D\left(W_{n, 2}\right)$ \\
\hline$n=3$ & 116 & 0.763158 & 6518 & 0.763232 & 74760 & 0.777196 \\
\hline$n=4$ & 444 & 0.792857 & 74597 & 0.794659 & 1701059 & 0.800906 \\
\hline$n=5$ & 1617 & 0.811747 & 825165 & 0.802752 & 37355674 & 0.804942 \\
\hline$n=6$ & 5789 & 0.821251 & 8896844 & 0.803639 & 792991830 & 0.80494 \\
\hline$n=7$ & 20519 & 0.824719 & 93866421 & 0.802885 & 16393751516 & 0.804318 \\
\hline$n=8$ & 72064 & 0.825135 & 973031117 & 0.801938 & 332260056199 & 0.803706 \\
\hline$n=9$ & 250841 & 0.824294 & 9945496874 & 0.801099 & 6633082879524 & 0.803191 \\
\hline$n=10$ & 865923 & 0.823064 & 100509266345 & 0.800397 & 130855615202033 & 0.802768 \\
\hline
\end{tabular}

$P_{y}\left(W_{n, k}\right)$ stands for $\left.\left(\partial F\left(W_{n, k} ; y, 1\right) / \partial y\right)\right|_{y=1}(n=3,4, \ldots, 10 ; k=0,1,2)$.

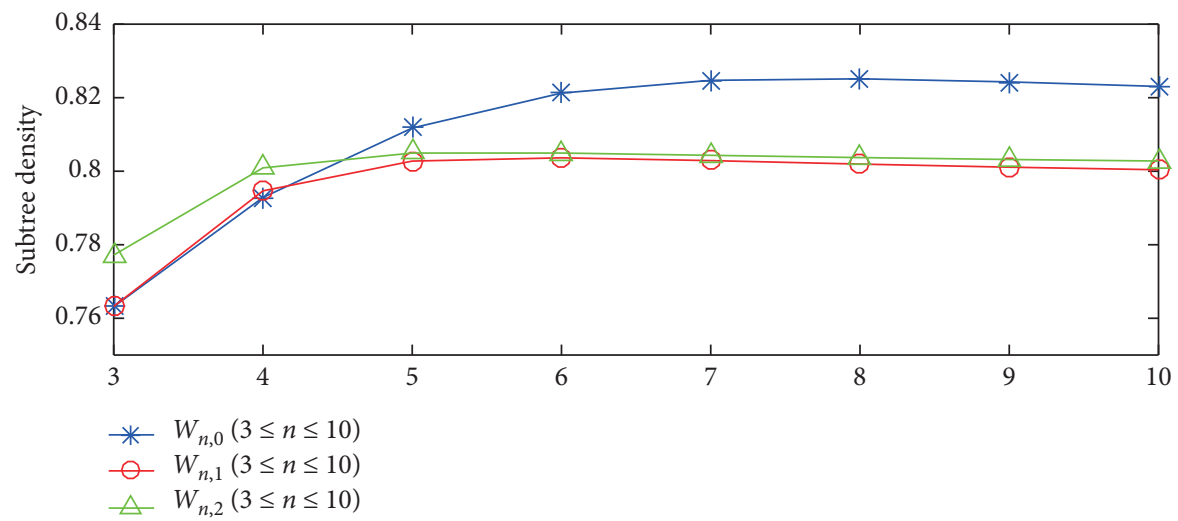

(a)

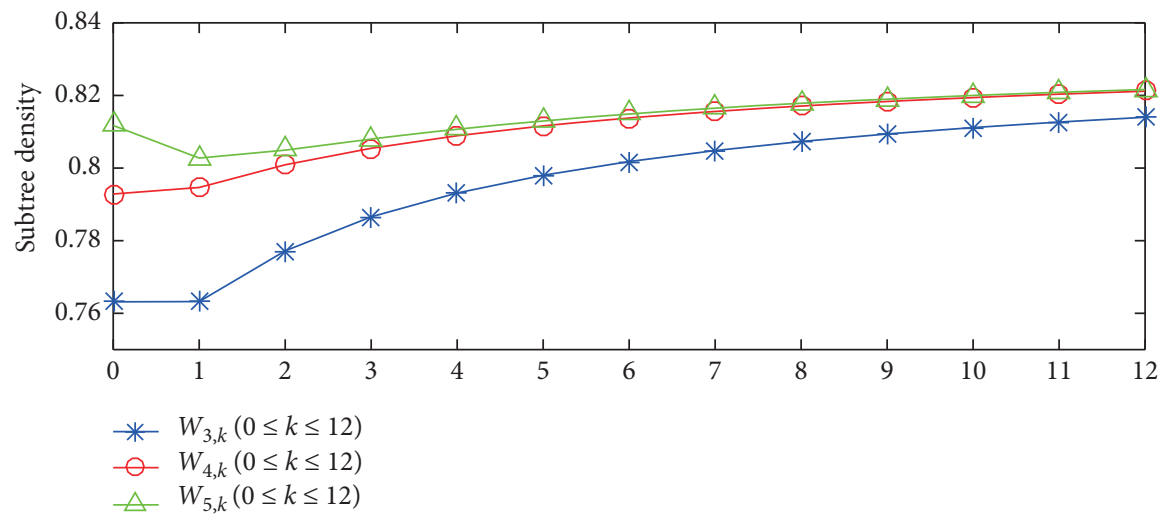

(b)

Figure 4: Subtree densities of $W_{n, k}(n=3,4, \ldots, 10 ; k=0,1, \ldots, 12)$. (a) Subtree densities of $W_{n, k}(n=3,4, \ldots, 10 ; k=0,1, \ldots, 12)$. (b) Subtree densities of $W_{n, k}(n=3,4,5 ; k=0,1, \ldots, 12)$.

increases with the increase of $n$. As illustrated in Figure 3(b), when $n \in\{3,4,5\}$ is fixed, the subtree density of $F_{n, k}(0 \leq k \leq 15)$ also increases with the increase of $k$.

4.3. Subtree Density of the $k$ Regular Wheel Graph. With Corollary 7 and equations (50) and (51), we can also obtain the subtree density of $W_{n, k}$ as shown in Table 6 and Figure 4; here, we skip the repetitive analysis details.

From Figure $4(\mathrm{a})$, when $k \in\{0,1,2\}$ is fixed, we notice a very interesting observation: the subtree density of
$W_{n, k}(3 \leq n \leq 10)$ gradually decreases with the increase of $n$. This trend needs to be further verified through combinatoric approaches. When $n \in\{3,4,5\}$ is fixed, by observing Figure $4(\mathrm{~b})$, it appears that the subtree density of $W_{n, k}(0 \leq k \leq 12)$ increases with the increase of $k$.

\section{Conclusion}

As an important topological index of graphs and networks, the subtree number index is closely related to the analysis of the hybrid local reliable network and discovery of new 
properties of compounds. We present the subtree generating functions and subtree number indices of the generalized book graph $B(n)(n \geq 2)$, generalized fan graph $F(n)(n \geq 3)$, and generalized wheel graph $W(n)(n \geq 3)$. Furthermore, we also briefly discuss the subtree density asymptotic characteristics of the $k$ regular cases of these three graphs: $B_{n, k}, F_{n, k}$, and $W_{n, k}$. Our study provides some basis for exploring hidden properties of complex cyclic graphs and chemical compounds from a new metric.

\section{Data Availability}

The data used to support the findings of this study are available from the corresponding author upon request.

\section{Conflicts of Interest}

The authors declare that they have no conflicts of interest.

\section{Acknowledgments}

This study was supported by the National Natural Science Foundation of China (grant nos. 61702291 and 11801371), the Program for Science \& Technology Innovation Talents in Universities of Henan Province (grant no. 19HASTIT029), the Key Research Project in Universities of Henan Province (grant nos. 19B110011 and 19B630015), and the Scientific Research Starting Foundation for High-Level Talents of Pingdingshan University (grant no. PXY-BSQD2017006).

\section{References}

[1] K. C. Das, J. M. Rodríguez, and J. M. Sigarreta, "On the maximal general abc index of graphs with given maximum degree," Applied Mathematics and Computation, vol. 386, Article ID 125531, 2020.

[2] E. Estrada, "Generalization of topological indices," Chemical Physics Letters, vol. 336, no. 3-4, pp. 248-252, 2001.

[3] A. Hussain, M. Numan, N. Naz, S. I. Butt, A. Aslam, and A. Fahad, "On topological indices for new classes of benes network," Journal of Mathematics, vol. 2021, Article ID 6690053, 7 pages, 2021.

[4] G. Wang and Y. Liu, "The edge-wiener index of zigzag nanotubes," Applied Mathematics and Computation, vol. 377, p. 125191, 2020.

[5] A. A. Dobrynin, R. Entringer, and I. Gutman, "Wiener index of trees: theory and applications," Acta Applicandae Mathematicae, vol. 66, no. 3, pp. 211-249, 2001.

[6] H. Wiener, "Structural determination of paraffin boiling points," Journal of the American Chemical Society, vol. 69, no. 1, pp. 17-20, 1947.

[7] R. E. Merrifield and H. E. Simmons, Topological Methods in Chemistry, Wiley, New York, NY, USA, 1989.

[8] L. A. Székely and H. Wang, "On subtrees of trees," Advances in Applied Mathematics, vol. 34, no. 1, pp. 138-155, 2005.

[9] B. Knudsen, "Optimal multiple parsimony alignment with affine gap cost using a phylogenetic tree," in International Workshop on Algorithms in BioinformaticsSpringer, Berlin, Germany, 2003.

[10] Y. Xiao, H. Zhao, Z. Liu, and Y. Mao, "Trees with large numbers of subtrees," International Journal of Computer Mathematics, vol. 94, no. 2, pp. 372-385, 2017.
[11] P. Welke, T. Horváth, and S. Wrobel, "Probabilistic and exact frequent subtree mining in graphs beyond forests," Machine Learning, vol. 108, no. 7, pp. 1137-1164, 2019.

[12] R. E. Jamison, "On the average number of nodes in a subtree of a tree," Journal of Combinatorial Theory, Series B, vol. 35, no. 3, pp. 207-223, 1983.

[13] R. E. Jamison, "Monotonicity of the mean order of subtrees," Journal of Combinatorial Theory, Series B, vol. 37, no. 1, pp. 70-78, 1984.

[14] W. Yan and Y.-N. Yeh, "Enumeration of subtrees of trees," Theoretical Computer Science, vol. 369, no. 1-3, pp. 256-268, 2006.

[15] Y. Yang, H. Liu, H. Wang, A. Deng, and C. Magnant, "On algorithms for enumerating subtrees of hexagonal and phenylene chains," The Computer Journal, vol. 60, pp. 690-710, 2017.

[16] Y. Yang, H. Liu, H. Wang, and H. Fu, "Subtrees of spiro and polyphenyl hexagonal chains," Applied Mathematics and Computation, vol. 268, pp. 547-560, 2015.

[17] Y. Yang, X.-J. Sun, J.-Y. Cao, H. Wang, and X.-D. Zhang, “The expected subtree number index in random polyphenylene and spiro chains," Discrete Applied Mathematics, vol. 285, pp. 483-492, 2020.

[18] A. J. Chin, G. Gordon, K. J. MacPhee, and C. Vincent, "Subtrees of graphs," Journal of Graph Theory, vol. 89, no. 4, pp. 413-438, 2018.

[19] S. Li, H. Wang, and S. Wang, "Some extremal ratios of the distance and subtree problems in binary trees," Applied Mathematics and Computation, vol. 361, pp. 232-245, 2019.

[20] B. Kamiński and P. Prałat, "Sub-trees of a random tree," Discrete Applied Mathematics, vol. 268, pp. 119-129, 2019.

[21] X.-M. Zhang, H. Wang, and X.-D. Zhang, "On the eccentric subtree number in trees," Discrete Applied Mathematics, vol. 290, pp. 123-132, 2021.

[22] Y. Lin, J. Shu, and Y. Meng, "Laplacian spectrum characterization of extensions of vertices of wheel graphs and multifan graphs," Computers \& Mathematics with Applications, vol. 60, no. 7, pp. 2003-2008, 2010.

[23] S. N. Daoud, "Complexity of graphs generated by wheel graph and their asymptotic limits," Journal of the Egyptian Mathematical Society, vol. 25, no. 4, pp. 424-433, 2017.

[24] Y. Yang, A. Wang, H. Wang, W.-T. Zhao, and D.-Q. Sun, “On subtrees of fan graphs, wheel graphs, and "partitions" of wheel graphs under dynamic evolution," Mathematics, vol. 7, no. 5, p. 472, 2019.

[25] F. Barioli, "Completely positive matrices with a book-graph," Linear Algebra and Its Applications, vol. 277, no. 1-3, pp. 11-31, 1998.

[26] X. Yu, L. Zhou, and X. Li, "A novel hybrid localization scheme for deep mine based on wheel graph and chicken swarm optimization," Computer Networks, vol. 154, pp. 73-78, 2019.

[27] X. Liu, Y. Zhang, and X. Gui, "The multi-fan graphs are determined by their laplacian spectra," Discrete Mathematics, vol. 308, no. 18, pp. 4267-4271, 2008. 